\title{
Universality of affine formulation in General Relativity theory
}

\author{
Jerzy Kijowski \\ and \\ Roman Werpachowski \\ Center for Theoretical Physics, Polish Academy of Sciences \\ e-mail: roman@cft.edu.pl
}

5.I.2007

\begin{abstract}
The affine variational principle for General Relativity, proposed in 1978 by one of us, is a good remedy for the nonuniversal properties of the standard, metric formulation, arising when the matter Lagrangian depends upon the metric derivatives. The affine version of the theory cures the standard drawback of the metric version, where the leading (second order) term of the field equations depends upon the matter fields and its causal structure violates the light cone structure of the metric. Choosing the affine connection (and not the metric one) as the gravitational configuration, simplifies considerably the canonical structure of the theory and is more suitable for the purposes of its quantization along the lines of Ashtekar and Lewandowski. We show how the affine formulation provides a simple method to handle boundary integrals in General Relativity theory.
\end{abstract}

Keywords: variational principles, affine principle, affine connection.

\section{Introduction}

The field equations of electrodynamics may be written in a universal form: $F=d A$ and $d \mathcal{F}=\mathcal{J}$, valid also in the case of nonlinear models. The only difference between the linear (Maxwell) theory and its nonlinear variants (Mie, Born-Infeld, phenomenological models of continuous media etc.) consists in the constitutive equation, relating the electromagnetic tensor $F$ (encoding fields $E$ and $B$ ) with the "induction tensor" $\mathcal{F}$ (encoding fields $D$ and $H$ ): the Maxwell constitutive equation $\mathcal{F}=* F$ is no longer valid in the nonlinear cases. 
Einstein's theory of gravity is often interpreted in a similar way: the metric connection $\Gamma$ expressed in terms of derivatives of the metric plays role of the homogeneous equation $F=d A$, whereas the Einstein equations $\mathcal{G}=\kappa \mathcal{T}$ correspond to the nonhomogeneous Maxwell equation. One argues that the Einstein tensor $\mathcal{G}$ is a universal differential operator acting on the metric, whereas the matter energy-momentum tensor $\mathcal{T}$ plays role of the "source" and depends upon the specific matter properties, according to the "constitutive equations":

$$
\mathcal{T}^{\mu \nu}:=2 \frac{\delta \mathcal{L}_{\mathrm{M}}^{\mathrm{mat}}}{\delta g_{\mu \nu}}=2\left(\frac{\partial \mathcal{L}_{\mathrm{M}}^{\mathrm{mat}}}{\partial g_{\mu \nu}}-\partial_{\lambda} \frac{\partial \mathcal{L}_{\mathrm{M}}^{\mathrm{mat}}}{\partial g_{\mu \nu, \lambda}}\right) .
$$

Such an interpretation fails, however, when the matter Lagrangian $\mathcal{L}_{\mathrm{M}}^{\text {mat }}$ depends upon the derivatives $\partial g$ of the metric via the Christoffel symbols, contained e. g. in the covariant derivatives of the matter fields. In this case, the last term of (11) contains the second derivatives of the metric. Hence, the Einstein equations $\mathcal{G}=\kappa \mathcal{T}$ imply that the second order differential operator acting on the metric is composed of two parts: 1) the universal part coming from $\mathcal{G}$ which, as is generally known, respects the light-cone structure of the metric $g$ and 2) the second order terms contained in $\mathcal{T}$. The causal structure of the resulting operator depends, therefore, upon the matter configuration and may differ considerably from the light-cone structure of the metric. This means that the small perturbations of a solution could, possibly, propagate outside of the light-cone (see Section 4 for a more detailed discussion of these nonuniversal features).

In the present paper we show that it is possible to restore the universality of the theory by rewriting it in the so-called affine formulation. It is based on the "affine variational principle" for General Relativity, proposed by one of us (J.K.) in 1978 [1]. It consists in deriving the Einstein theory of the combined "gravity + matter" system from the first order Lagrangian density 1

$$
\mathcal{L}_{\mathrm{A}}=\mathcal{L}_{\mathrm{A}}(\Gamma, \partial \Gamma, \varphi, \partial \varphi),
$$

where $\Gamma$ is an arbitrary symmetric space-time connection: $\Gamma=\left(\Gamma_{\mu \nu}^{\lambda}\right)$ with $\Gamma_{\mu \nu}^{\lambda}=\Gamma_{\nu \mu}^{\lambda}$, and $\varphi$ stands for matter field(s) of any nature. Physically, $\Gamma$ describes the local inertial frames which are necessary for the Einstein's interpretation of gravity in terms of the "freely falling elevators". Indeed, Einstein's approach to gravity is based on the following observation: at each space-time point $p$ the gravitational force may be eliminated and the Newton equation for freely falling test bodies may be reduced to $\ddot{y}^{k}(t)=0$. The possibility of such an elimination is equivalent to the existence of a "local inertial frame" at each point $p$ separately (global inertial frames do not exist

\footnotetext{
${ }^{1}$ In General Relativity one always deals with Lagrangian densities and not with scalars, since there is no volume form given a priori. To simplify the terminology, we will omit the word "density" when discussing Lagrangians.
} 
in general). Mathematically, such a frame may be defined as an equivalence class of local coordinate charts. The equivalence is understood with respect to the following relation: two charts $\left(y^{\alpha}\right)$ and $\left(x^{\mu}\right)$ are equivalent (belong to the same class) if their mutual second derivatives $\frac{\partial^{2} y^{\alpha}}{\partial x^{\mu} \partial x^{\nu}}$ vanish at $p$. Gravitational field may thus be described as a field of such local inertial frames.

For analytical purposes, every local inertial frame at $p$ may be uniquely characterized with respect to a given generic (not necessarily inertial!) reference chart $\left(x^{\mu}\right)$, by combinations of derivatives

$$
\Gamma_{\mu \nu}^{\lambda}(p):=\frac{\partial x^{\lambda}}{\partial y^{\alpha}} \frac{\partial^{2} y^{\alpha}}{\partial x^{\mu} \partial x^{\nu}}(p)
$$

of its arbitrary representative $\left(y^{\alpha}\right)$. It is easy to see that the components $\Gamma$ do not depend upon the choice of a representative $\left(y^{\alpha}\right)$ of the given equivalence class which constitutes the inertial frame in question. Moreover, they transform like components of a symmetric connection with respect to changes of the chart $\left(x^{\mu}\right)$. The entire information about the local inertial frame may be retrieved from the $\Gamma$ 's: a coordinate chart belongs to the privileged equivalence class at $p$ if and only if the corresponding connection coefficients (3) vanish at $p$. The field $\Gamma$ captures entire information about the spacetime configuration of the gravitational field in the following sense: equation $\ddot{y}^{k}(t)=0$, written in the inertial frame, is equivalent to $\ddot{x}^{\lambda}+\Gamma^{\lambda}{ }_{\mu \nu} \dot{x}^{\mu} \dot{x}^{\nu}=0$ in an arbitrary frame. This implies that all the trajectories of the freely gravitating test bodies may be calculated once we know $\Gamma$. No information about the metric is necessary here.

The affine Lagrangian (2) contains no metric. However, every field configuration of this theory is also equipped with a metric tensor, carried through the momentum canonically conjugate to $\Gamma$ which, in turn, is defined via derivatives $\partial \mathcal{L}_{\mathrm{A}} / \partial(\partial \Gamma)$ of the Lagrangian with respect to partial derivatives of the configuration $\Gamma$. As will be seen in the sequel, it is natural to use the "covariant-density" version of the metric $(k=8 \pi G$ is the gravitational constant):

$$
\pi^{\mu \nu}:=\frac{\sqrt{|\operatorname{det} g|}}{2 k} g^{\mu \nu},
$$

and to relate it to the derivative of the Lagrangian with respect to a specific combination of derivatives $\partial_{\kappa} \Gamma^{\lambda}{ }_{\mu \nu}$, namely the one which is contained in the symmetric part $K_{\mu \nu}:=R_{(\mu \nu)}=\frac{1}{2}\left(R_{\mu \nu}+R_{\nu \mu}\right)$ of the Ricci tensor $R_{\nu \mu}$ of $\Gamma$ :

$$
\pi^{\mu \nu}=\frac{\partial \mathcal{L}_{\mathrm{A}}}{\partial K_{\mu \nu}} .
$$

Euler-Lagrange equations of this theory

$$
\frac{\delta \mathcal{L}_{\mathrm{A}}}{\delta \Gamma}=0, \quad \frac{\delta \mathcal{L}_{\mathrm{A}}}{\delta \varphi}=0,
$$


rewritten in terms of this metric, are equivalent to the conventional system: "Einstein equations + matter equations". Of course, only solutions with the correct signature $(-,+,+,+)$ describe physical spacetime geometries. This additional restriction on the solutions of the above field equations must, therefore, be imposed (see also discussion in Section 3, below formula (41)). We stress, however, that for many purposes, like the string theory or the "complex heaven" theory, different signatures or even a complexification of Einstein theory might also be interesting [2, 3]. Within this (already classical) framework, a restriction on the signature of the metric is thus also necessary in order to select physically admissible solutions among all possible mathematical solutions of the theory. As we show in the sequel, the affine formulation does not rely on the specific signature of the metric and exists in all these cases.

As an example take the following affine Lagrangian, depending upon the connection, the scalar matter field $\phi$ and their derivatives:

$$
\mathcal{L}_{\mathrm{A}}(K, \phi, \partial \phi)=\frac{2}{m^{2} \phi^{2}} \sqrt{\left|\operatorname{det}\left(\frac{1}{k} K_{\mu \nu}-\phi_{, \mu} \phi_{, \nu}\right)\right|} .
$$

Definition (5) of the canonical momenta $\pi$ implies the following relation between the metric and the curvature:

$$
\pi^{\mu \nu}=\frac{1}{m^{2} \phi^{2}} \sqrt{\left|\operatorname{det}\left(\frac{1}{k} K_{\mu \nu}-\phi_{, \mu} \phi_{, \nu}\right)\right|}\left[\left(\frac{1}{k} K-\partial \phi \cdot \partial \phi\right)^{-1}\right]^{\mu \nu},
$$

where $\left(\frac{1}{k} K+\partial \phi \cdot \partial \phi\right)^{-1}$ is the matrix inverse of $\left(\frac{1}{k} K_{\mu \nu}-\phi_{, \mu} \phi_{, \nu}\right)$. Calculating the determinant of both sides and using definition (4), we may obtain the following metric tensor

$$
g_{\mu \nu}=\frac{2}{m^{2} \phi^{2}}\left(\frac{1}{k} K_{\mu \nu}-\phi_{, \mu} \phi_{, \nu}\right)
$$

and, consequently, we have:

$$
K_{\mu \nu}=\frac{k}{2} m^{2} \phi^{2} g_{\mu \nu}+k \phi_{, \mu} \phi_{, \nu}
$$

Einstein tensor $G_{\mu \nu}=K_{\mu \nu}-\frac{1}{2} K_{\alpha \beta} g^{\alpha \beta} g_{\mu \nu}$ fulfils, therefore, the following equations:

$$
\frac{1}{k} G_{\mu \nu}=\left(\phi_{, \mu} \phi_{, \nu}-\frac{1}{2} m^{2} \phi^{2} g_{\mu \nu}-g^{\alpha \beta} \phi_{, \alpha} \phi_{, \beta} g_{\mu \nu}\right) .
$$

These are Einstein equations $G=k T$ for the Klein-Gordon scalar field, whose energy-momentum tensor $T_{\mu \nu}$ is described by the right-hand side of the above equation. It will be proved in the sequel (see Section 3) that: 
1. The first Euler-Lagrange equations in (6) implies that $\Gamma$ is the metric (Levi-Civitta) connection of $g$ (and, whence, $K_{\mu \nu}=R_{\mu \nu}$ ),

2. The second Euler-Lagrange equations in (6) implies the Klein-Gordon equation

$$
\left(\square-m^{2}\right) \phi=0,
$$

where $\square$ is the natural d'Alembert operator for the metric $g$.

We conclude that (77) is the affine Lagrangian of the Einstein-Klein-Gordon theory.

Even if perfectly equivalent to the conventional (metric) formulation of the General Relativity theory, the new (affine) formulation clarifies considerably its canonical structure. This is due to the fact that all the boundary integrals, otherwise very complicated and even messy, gather in a natural way to the integral of the affine contact form " $\pi \cdot \delta \Gamma$ " (analogous to " $p \cdot \delta q$ " in canonical mechanics). This observation simplifies enormously any manipulation of boundary terms in General Relativity, regardless of which of the three variational principles one begins with: 1) metric, 2) affine or 3) metric-affine (Palatini). As an application, one obtains an effective criterion which enables us to check which one among the many existing quasi-local expressions for the gravitational mass really corresponds to the field hamiltonian [4, 5, 6]. Recently, this technique was used to identify the first law of thermodynamics of black holes with the energy flux due to the variation of the field boundary data, and to generalize it to dynamical black holes [7]. A similar integration of the " $\pi \cdot \delta \Gamma$ " term over a wave front gives the simplest derivation of the dynamics of a null-like, self-gravitating shell [8]. Recently, the same techniques was used to derive the variational principle for the "spherically symmetric massive shell + gravity" system [9].

The affine point of view is confirmed by the recent developments of the quantum gravity [10]. Indeed, the fundamental idea of the Ashtekar description of the canonical structure of the space of the Cauchy data for the Einstein equations is that the field configuration is encoded in the connection, whereas the metric plays role of a corresponding momentum (see e.g. [11, 12]). This picture is much more natural when we construct the canonical structure already from the affine variational principle [4].

The affine picture in the General Relativity theory should also be taken seriously into account when we try to quantize gravity via path integrals (the so-called "quantum foam" approach). Interpreting the scalar curvature not as a degenerate Lagrangian (degenerate, because linear in highest derivatives!) but merely as a standard , $p \cdot \dot{q}$ " term in the Legendre transformation formula , $L=p \cdot \dot{q}-H$ " (see formula (29)) changes completely the philosophy of path integrals, where the integration should be performed over the space of connections $\Gamma$ rather than over the space of metrics. This 
result strongly supports the Ashtekar-Lewandowski approach to Quantum Gravity.

Our paper is organized as follows. In Section 2 we illustrate the relations between various variational principles on a toy model of classical mechanics. In Section 3 we briefly sketch the results of paper [1] and give a simple argument for the validity of the affine picture in a simplest case, when the matter Lagrangian does not depend upon the derivatives of the metric, i. e. when the last (nonuniversal) term in (11) vanishes. Section 4 deals already with generic Lagrangians. We construct a certain nonmetric connection $\Gamma$ and show that the Einstein equations recover their universality when rewritten in terms of $\Gamma$. In Section 5 we prove that also the matter field equations are equivalent in both the metric and the affine picture. Mathematically, these results are equivalent to those proved in [13] and [21]. However, the approach proposed here is better adapted for the purposes of the metric theories and the proofs are new. Section 6 contains a brief summary of the particular features of the affine approach. Finally, Appendix A shows how to handle in a simple way the boundary terms in GR. The method is based on the affine picture, but its validity is universal. One may apply it to Lagrangian or Hamiltonian problems, whenever the contribution from the boundary has to be taken into account [4, 7, 8, 9, 14. Technical proofs have been shifted to Appendix B. We have provided an example of the transformation between the affine and metric principles in Appendix $\mathrm{C}$

\section{A heuristic idea of the affine formulation}

To illustrate the relation between the metric and the affine formulation, we consider a toy example: the one-dimensional harmonic oscillator. It is described by two quantities: the momentum $p$ and the configuration $q$. They fulfill the following dynamical equations:

$$
\begin{aligned}
& p=m \dot{q}, \\
& \dot{p}=-k q .
\end{aligned}
$$

As has been noticed in [15], the theory may be derived from the following second order Lagrangian

$$
L_{\mathrm{M}}(p, \dot{p}, \ddot{p})=-\frac{1}{k} p \ddot{p}-\frac{1}{2 k} \dot{p}^{2}-\frac{1}{2 m} p^{2},
$$

if we keep (14) as a definition of an "auxiliary variable"

$$
q:=-\frac{1}{k} \dot{p} .
$$


Indeed, variation with respect to $p$ reproduces the remaining equation (13):

$$
\begin{aligned}
\frac{\delta L_{\mathrm{M}}}{\delta p} & =\frac{\mathrm{d}^{2}}{\mathrm{~d} t^{2}} \frac{\partial L_{\mathrm{M}}}{\partial \ddot{p}}-\frac{\mathrm{d}}{\mathrm{d} t} \frac{\partial L_{\mathrm{M}}}{\partial \dot{p}}+\frac{\partial L_{\mathrm{M}}}{\partial p} \\
& =-\frac{1}{k} \ddot{p}-\frac{1}{m} p=\dot{q}-\frac{1}{m} p=0 .
\end{aligned}
$$

The above "crazy" formulation is very much analogous to the metric formulation of relativity, where $p$ and $q$ play the roles of the metric $g$ and the metric connection $\Gamma$ respectively. Like the Hilbert Lagrangian in the gravity theory, the quantity $L_{\mathrm{M}}$ is linear with respect to the second derivatives and quadratic with respect to the first derivatives of the configuration $p$. The "Palatini method of variation" consists in replacing the first derivatives of the configuration ( $\dot{p}$ here and $\partial g$ in the gravity theory) by "an auxiliary variable" ( $q$ here and $\Gamma$ in the gravity theory). This leads to the following "Palatini version" of (15):

$$
L_{\mathrm{P}}(p, q, \dot{q})=p \dot{q}-\frac{k}{2} q^{2}-\frac{1}{2 m} p^{2} .
$$

Here, the variation with respect to $q$ reproduces its definition (16), whereas the variation with respect to $p$ reproduces the dynamical equation (17) (analogous to the Einstein equation in the gravity theory).

Finally, using the latter equation, we may eliminate $p$ (the metric) from the Lagrangian and obtain its "affine version" which turns out to be the conventional Lagrangian for the oscillator:

$$
L_{\mathrm{A}}(q, \dot{q})=\frac{m}{2} \dot{q}^{2}-\frac{k}{2} q^{2},
$$

numerically equal to the previous Lagrangians $L_{\mathrm{M}}$ and $L_{\mathrm{P}}$. The above quantity $L_{\mathrm{A}}$ is as an analogue of the affine Lagrangian in the gravity theory. Now, (14) is treated as the dynamical equation, which may be derived from the Lagrangian $L_{\mathrm{A}}$, whereas (13) is merely a definition of an auxiliary variable $p$.

The standard canonical structure of the theory based on $L_{A}$ is fully given by the symplectic form $\mathrm{d} p \wedge \mathrm{d} q$. Its derivation is very much analogous to the canonical gravity derived from the affine formulation (see [4, 16]). The derivation of this structure from the original second order Lagrangian (15) or its "Palatini version" (18) is much more difficult and leads basically to second-type constraints. We recover the final result only when we reduce the theory with respect to these constraints. Such a procedure is very much analogous to the original ADM procedure [17] in the gravity theory, whereas the ,affine" Lagrangian (19) leads directly to the correct canonical structure of the theory. 


\section{A simple argument for the affine formulation of Einstein gravity theory}

It was proved in [1] that the Euler-Lagrange equations (6) are equivalent to the conventional system "the Einstein equations + the matter equations" provided the following condition is satisfied:

$\mathcal{L}_{\mathrm{A}}$ is an invariant scalar density which depends upon a connection $\Gamma$ and its derivatives only via the symmetric part $K_{\mu \nu}:=R_{(\mu \nu)}=\frac{1}{2}\left(R_{\mu \nu}+R_{\nu \mu}\right)$ of the Ricci tensor $R_{\nu \mu}$ of $\Gamma$ :

$$
\mathcal{L}_{\mathrm{A}}=\mathcal{L}_{\mathrm{A}}(K, \varphi, \partial \varphi) .
$$

No metricity condition is imposed on $\Gamma$, because there is no metric in $\mathcal{L}_{\mathrm{A}}$. This is why the Ricci tensor could possess a priori an anti-symmetric part. However, any field configuration of this theory carries also a metric tensor $g=\left(g_{\mu \nu}\right)$, defined in its "contravariant-density" version (44) by the following equations:

$$
\pi^{\mu \nu}:=\frac{\partial \mathcal{L}_{\mathrm{A}}(K, \varphi, \partial \varphi)}{\partial K_{\mu \nu}}
$$

The equivalence of the two theories means that, when rewritten in terms of this metric tensor, the field equations (6) are equivalent to the combined "Einstein equations + matter field equations" for a certain matter theory, whose matter Lagrangian is uniquely constructed in terms of the affine Lagrangian $\mathcal{L}_{\mathrm{A}}$.

A simple argument given in [1] for the equivalence of the above "affine theory" and the conventional metric formulation of the General Relativity theory follows from the observation that the gravitational part of the EulerLagrange equations (6) may be written as follows:

$$
0=-\frac{\delta \mathcal{L}_{\mathrm{A}}}{\delta \Gamma_{\mu \nu}^{\lambda}}=\partial_{\kappa}\left\{\frac{\partial \mathcal{L}_{\mathrm{A}}}{\partial K_{\alpha \beta}} \frac{\partial K_{\alpha \beta}}{\partial \Gamma_{\mu \nu, \kappa}^{\lambda}}\right\}-\frac{\partial \mathcal{L}_{\mathrm{A}}}{\partial K_{\alpha \beta}} \frac{\partial K_{\alpha \beta}}{\partial \Gamma_{\mu \nu}^{\lambda}},
$$

where we denote

$$
\Gamma_{\mu \nu, \kappa}^{\lambda}:=\partial_{\kappa} \Gamma_{\mu \nu}^{\lambda} .
$$

The definition of the Ricci tensor

$$
K_{\mu \nu}:=\Gamma_{\mu \nu, \lambda}^{\lambda}-\Gamma_{(\mu|\lambda|, \nu)}^{\lambda}+\Gamma_{\alpha \lambda}^{\lambda} \Gamma_{\mu \nu}^{\alpha}-\Gamma_{\alpha \nu}^{\lambda} \Gamma_{\mu \lambda}^{\alpha}
$$

implies that $K$ is linear in $\Gamma_{\mu \nu, \kappa}^{\lambda}$ and quadratic in $\Gamma_{\mu \nu}^{\lambda}$. Hence, we end up with an equation of the type

$$
\partial \pi-\Gamma \cdot \pi=0 .
$$

As will be seen in the sequel (see Appendix (A), the term " $\Gamma \cdot \pi$ " in (24) is precisely what we need to convert the first term " $\partial \pi$ " into the covariant 
derivative $\nabla \pi$ with respect to $\Gamma$. Finally, the gravitational part (22) of the Euler-Lagrange equations (6) reads: $\nabla_{\lambda} \pi^{\mu \nu}=0$ or, equivalently,

$$
\nabla_{\lambda} g_{\mu \nu}=0
$$

which implies that $\Gamma$ must be equal to the Levi-Civita (metric) connection of $g$. Metricity condition for the connection (not postulated a priori) is, therefore, derived from the Lagrangian as the field dynamics (cf. (14) in our toy model). On the other hand, Einstein equations, treated in the conventional approach as field dynamics, are derived here a priori from the Lagrangian as the constitutive relation (21) between the matter fields and the Ricci curvature $R_{\mu \nu}$ (which, due to the metricity condition (25), reduces to $K_{\mu \nu}$ ) - cf. (13) in the toy model.

To analyze also the matter dynamics $\frac{\delta \mathcal{L}_{\mathrm{A}}}{\delta \varphi}=0$, it is convenient to perform the following Legendre transformation:

$$
\mathcal{L}_{\mathrm{A}}^{\mathrm{mat}}(\pi, \varphi, \partial \varphi):=\mathcal{L}_{\mathrm{A}}(K, \varphi, \partial \varphi)-\pi^{\mu \nu} K_{\mu \nu},
$$

where $K=K(\pi, \varphi, \partial \varphi)$ on the right-hand side are just the "Einstein equations", obtained by solving algebraically equations (21) with respect to $K$ (similarly as it was done in (10) for the Einstein-Klein-Gordon case). It is a standard property of the Legendre transformation between mutually conjugate objects $K_{\mu \nu}$ and $\pi^{\mu \nu}$, that equation $\pi=\partial \mathcal{L}_{\mathrm{A}} / \partial K$ is converted 2 into equation $K=-\partial \mathcal{L}_{\mathrm{A}}^{\mathrm{mat}} / \partial \pi$. Hence, equations (21) are equivalent to

$$
K_{\mu \nu}=-\frac{\partial \mathcal{L}_{\mathrm{A}}^{\mathrm{mat}}}{\partial \pi^{\mu \nu}}
$$

Moreover, the equivalence

$$
\left(\frac{\delta \mathcal{L}_{\mathrm{A}}}{\delta \varphi}=0\right) \Longleftrightarrow\left(\frac{\delta \mathcal{L}_{\mathrm{A}}^{\mathrm{mat}}}{\delta \varphi}=0\right)
$$

may be easily proved (see [1] or Appendix [A). This means that $\mathcal{L}_{\mathrm{A}}^{\text {mat }}$ obtained from (26) plays role of the matter Lagrangian for the field $\varphi$. According to (26) the numerical value of the affine Lagrangian equals

$$
\mathcal{L}_{\mathrm{A}}=\mathcal{L}_{\mathrm{A}}^{\text {mat }}+\pi^{\mu \nu} K_{\mu \nu}=\mathcal{L}_{\mathrm{A}}^{\text {mat }}+\frac{\sqrt{|\operatorname{det} g|}}{2 k} g^{\mu \nu} R_{\mu \nu},
$$

and, whence, coincides with the conventional Hilbert Lagrangian, with the metric tensor eliminated by the means of relation (21).

The following examples of the affine Lagrangians were analyzed in detail:

\footnotetext{
${ }^{2}$ In classical mechanics equation $p=\partial L / \partial \dot{q}$ converts to equation $-\dot{q}=\partial(-H) / \partial p$, where $-H=L-p \dot{q}$.
} 
1. A general nonlinear Einstein-Klein-Gordon theory [1]:

$$
\mathcal{L}_{\mathrm{A}}(K, \phi, \partial \phi)=\frac{1}{U(\phi)} \sqrt{\left|\operatorname{det}\left(\frac{1}{k} K_{\mu \nu}-\phi_{, \mu} \phi_{, \nu}\right)\right|},
$$

where $\phi_{, \mu}=\partial_{\mu} \phi$ and $U(\phi)$ is a scalar function. In particular $U(\phi)=$ $\frac{1}{2} m^{2} \phi^{2}$ for the linear Klein-Gordon theory, cf. (7).

2. The Einstein-Maxwell theory [15]

$$
\mathcal{L}_{\mathrm{A}}(K, \partial A)=-\frac{1}{4} \sqrt{\left|\operatorname{det} K_{\rho \sigma}\right|} K^{\mu \nu} K^{\alpha \beta} F_{\mu \alpha} F_{\nu \beta},
$$

where $A_{\mu}$ is the electromagnetic potential of the electromagnetic field $F_{\mu \nu}=\partial_{\mu} A_{\nu}-\partial_{\nu} A_{\mu}$, whereas $K^{\mu \nu}$ is the contravariant tensor, inverse to $K_{\alpha \beta}$ (i. e. defined by: $K^{\mu \alpha} K_{\alpha \beta}=\delta_{\beta}^{\mu}$ ).

3. The vacuum Einstein theory:

$$
\mathcal{L}_{\mathrm{A}}(K)=-\frac{1}{k \Lambda} \sqrt{|\operatorname{det} K|},
$$

where $\Lambda$ is the cosmological constant [15].

4. The Einstein-Euler equations describing a self-gravitating barotropic fluid:

$$
\mathcal{L}_{\mathrm{A}}(K, \xi, \partial \xi)=-\sqrt{|\operatorname{det} K|} f\left(\frac{K_{\mu \nu} j^{\mu} j^{\nu}}{\operatorname{det} K}\right),
$$

where the function $f$ uniquely implies the constitutive equation of the fluid [19] and $j$ is the vector density representing the matter current of the fluid. The latter is uniquely defined by the fluid configuration $\xi$ and its partial derivatives, without any knowledge of the metric (see [14]). In particular, the linear function $f(y)=\frac{k}{8} y$ corresponds to the case of a dust (whose pressure vanishes identically: $p \equiv 0$ ).

The following observation concerning these models seems to hold universally: "what is simple in the metric picture becomes, in general, complicated in the affine picture and vice versa". This may be a good leading principle in the search for fundamental, unified theories of various interactions. Indeed, the simplicity of the Lagrangian is an important feature of fundamental theories. For the gravitational interaction, affine approach looks much more fundamental than the metric approach.

Each of the affine theories (30)-(33) is characterized by the fact that, in the conventional metric formulation, its matter Lagrangian $\mathcal{L}_{\mathrm{M}}^{\text {mat }}$ does not contain the derivatives of the metric tensor because no "covariantization" of the partial derivatives of the matter field is necessary:

$$
\mathcal{L}_{\mathrm{M}}^{\mathrm{mat}}=\mathcal{L}_{\mathrm{M}}^{\mathrm{mat}}(g, \varphi, \partial \varphi) .
$$


To derive the affine formulation for the matter fields of this type, it is useful to rewrite the conventional Einstein equations in a simpler form, analogous to (27) 3 :

$$
\left(\stackrel{\circ}{K}_{\mu \nu}=\right) \stackrel{\circ}{R}_{\mu \nu}=-\frac{\partial \mathcal{L}_{\mathrm{M}}^{\mathrm{mat}}}{\partial \pi^{\mu \nu}} .
$$

Indeed, let us take the standard metric Lagrangian $\mathcal{L}_{\mathrm{M}}=\mathcal{L}_{\mathrm{H}}+\mathcal{L}_{\mathrm{M}}^{\text {mat }}$, where $\mathcal{L}_{\mathrm{H}}$ is the Hilbert Lagrangian

$$
\mathcal{L}_{\mathrm{H}}:=\frac{\sqrt{|\operatorname{det} g|}}{2 k} \stackrel{\circ}{R}=\pi^{\mu \nu} \stackrel{\circ}{K}_{\mu \nu} .
$$

The Einstein equations are derived as the Euler-Lagrange equations for $\mathcal{L}_{\mathrm{M}}$ :

$$
0=\frac{\delta \mathcal{L}_{\mathrm{M}}}{\delta g_{\mu \nu}}=-\frac{1}{2 k} \stackrel{\mathcal{G}}{\mu \nu}^{\mu \nu} \frac{\partial \mathcal{L}_{\mathrm{M}}^{\mathrm{mat}}(g, \varphi, \partial \varphi)}{\partial g_{\mu \nu}},
$$

because the variation of the Hilbert Lagrangian is proportional to the Einstein tensor density

$$
\stackrel{\mathcal{G}}{\mathcal{G}}^{\mu \nu}:=\sqrt{|\operatorname{det} g|}\left(\stackrel{\circ}{R}^{\mu \nu}-\frac{1}{2} \stackrel{\circ}{R} g^{\mu \nu}\right) .
$$

After substituting the variable $\pi$ instead of $g$, we obtain:

$$
\begin{aligned}
\stackrel{\mathcal{G}}{ }^{\mu \nu} & =2 k \frac{\partial \pi^{\rho \sigma}}{\partial g_{\mu \nu}} \frac{\partial \mathcal{L}_{\mathrm{M}}^{\mathrm{mat}}(\pi, \varphi, \partial \varphi)}{\partial \pi^{\rho \sigma}} \\
& =\sqrt{|\operatorname{det} g|}\left(g^{\mu \rho} g^{\nu \sigma}-\frac{1}{2} g^{\mu \nu} g^{\rho \sigma}\right) \frac{\partial \mathcal{L}_{\mathrm{M}}^{\mathrm{mat}}(\pi, \varphi, \partial \varphi)}{\partial \pi^{\rho \sigma}},
\end{aligned}
$$

which, after lowering the free indices, subtracting half of the trace and dividing by $\sqrt{|\operatorname{det} g|}$, gives precisely (35). By identifying the matter Lagrangians in both formulations:

$$
\mathcal{L}_{\mathrm{M}}^{\mathrm{mat}}(g, \varphi, \partial \varphi)=\mathcal{L}_{\mathrm{A}}^{\mathrm{mat}}(g, \varphi, \partial \varphi),
$$

we get (27), which proves that the theory may now be easily converted to the affine picture via the inverse Legendre transformation (29), where $g=g(K, \varphi, \partial \varphi)$ must be calculated as an implicit function from (35).

To illustrate the above issues, consider again the Einstein-Klein-Gordon theory. Its generalized (nonlinear) version is defined by the following metric matter Lagrangian:

$$
\begin{aligned}
\mathcal{L}_{\mathrm{M}}^{\mathrm{mat}} & =\sqrt{|\operatorname{det} g|}\left(-\frac{1}{2} g^{\mu \nu} \phi_{, \mu} \phi_{, \nu}-U(\phi)\right) \\
& =-k \pi^{\mu \nu} \phi_{, \mu} \phi_{, \nu}-4 k^{2} \sqrt{|\operatorname{det} \pi|} U(\phi),
\end{aligned}
$$

\footnotetext{
${ }^{3}$ By putting a circle “०” over a geometric object, we indicate its metricity. Hence, by $\stackrel{\circ}{\nabla}$ we mean the covariant derivative taken with respect to the metric connection $\stackrel{\circ}{\Gamma}$, $\stackrel{\circ}{\Gamma}^{\lambda}{ }_{\mu \nu} \equiv\left\{\begin{array}{l}\lambda \\ \mu \nu\end{array}\right\}$.
} 
where $U(\phi)$ is an arbitrary positive, convex function of $\phi$. In particular, $U(\phi)=\frac{1}{2} m^{2} \phi^{2}$ leads to the linear theory, governed by equation (11). We have used the formula $|\operatorname{det} g|=16 k^{4}|\operatorname{det} \pi|$, which is an immediate consequence of the definition (44) of $\pi$. According to formula (35) the Einstein equations may be written in the following form:

$$
K_{\mu \nu}=-\frac{\partial \mathcal{L}_{\mathrm{M}}^{\mathrm{mat}}}{\partial \pi^{\mu \nu}}=k \phi_{, \mu} \phi_{, \nu}+2 k^{2} U \sqrt{|\operatorname{det} \pi|}\left(\pi^{-1}\right)_{\mu \nu} .
$$

It enables us to calculate the metric tensor (the momentum canonically conjugate to $\Gamma)$ in terms of ,,velocities" $(K$ and $\partial \phi)$ :

$$
g_{\mu \nu}=2 k \sqrt{|\operatorname{det} \pi|}\left(\pi^{-1}\right)_{\mu \nu}=\frac{1}{U}\left(\frac{1}{k} K_{\mu \nu}-\phi_{, \mu} \phi_{, \nu}\right) .
$$

We see that the tensor $\frac{1}{k} K_{\mu \nu}-\phi_{, \mu} \phi_{, \nu}$ is proportional to the metric tensor. The physical signature requirement for $g$ implies that only those configurations $(\phi, \Gamma)$ for which $\left(\frac{1}{k} K_{\mu \nu}-\phi_{, \mu} \phi_{, \nu}\right)$ has the signature $(-,+,+,+)$ have to be taken into account.

To calculate the total affine Lagrangian of the theory, we perform the Legendre transformation:

$$
\begin{aligned}
\mathcal{L}_{\mathrm{A}} & =\mathcal{L}_{\mathrm{A}}^{\mathrm{mat}}+K_{\mu \nu} \pi^{\mu \nu}=\mathcal{L}_{\mathrm{A}}^{\mathrm{mat}}+k \pi^{\mu \nu} \phi_{, \mu} \phi_{, \nu}+8 k^{2} \sqrt{|\operatorname{det} \pi|} U(\phi) \\
& =4 k^{2} \sqrt{|\operatorname{det} \pi|} U(\phi) .
\end{aligned}
$$

Calculating the determinant of both sides of (41) gives the relation

$$
4 k^{2} U^{2} \sqrt{|\operatorname{det} \pi|}=\sqrt{\left|\operatorname{det}\left(\frac{1}{k} K_{\mu \nu}-\phi_{, \mu} \phi_{, \nu}\right)\right|} .
$$

Inserting it into (42) gives (30).

Unfortunately, this procedure does not work for general matter fields, where the matter Lagrangian contains covariant derivatives:

$$
\mathcal{L}_{\mathrm{M}}^{\mathrm{mat}}=\mathcal{L}_{\mathrm{M}}^{\mathrm{mat}}(g, \varphi, \stackrel{\circ}{\nabla} \varphi)=\mathcal{L}_{\mathrm{M}}^{\mathrm{mat}}(g, \stackrel{\circ}{\Gamma}, \varphi, \partial \varphi) .
$$

As an example we may consider the vector field $\varphi=\left(\varphi^{\mu}\right)$, which needs covariant derivatives $\left(\stackrel{\circ}{\nabla}_{\nu} \varphi^{\mu}=\partial_{\nu} \varphi^{\mu}+\stackrel{\circ}{\Gamma}_{\nu \lambda}^{\mu} \varphi^{\lambda}\right)$ to construct an invariant quantity depending on its first derivatives.

On the other hand, a general affine Lagrangian

$$
\mathcal{L}_{\mathrm{A}}=\mathcal{L}_{\mathrm{A}}(K, \varphi, \nabla \varphi)=\mathcal{L}_{\mathrm{A}}(K, \Gamma, \varphi, \partial \varphi)
$$

implies that the connection $\Gamma$ differs from the metric connection $\stackrel{\circ}{\Gamma}$, due to the nonhomogeneous term

$$
\mathcal{J}_{\lambda}^{\mu \nu}:=\frac{\partial \mathcal{L}_{\mathrm{A}}(K, \Gamma, \varphi, \partial \varphi)}{\partial \Gamma^{\lambda} \mu \nu},
$$


which arises on the right-hand side of (22). In fact, the gravitational part of the field equations (6) may now be rewritten (see [1] or Appendix A) as follows:

$$
\nabla_{\lambda} \pi^{\mu \nu}=\mathcal{J}_{\lambda}^{\mu \nu}-\frac{2}{3} \delta_{\lambda}^{(\mu} \mathcal{J}_{\rho}^{\nu) \rho}
$$

instead of (25). For a nonvanishing derivative (46), this implies $\Gamma \neq \stackrel{\circ}{\Gamma}$. Hence, the metric and the affine theories look quite different in a generic case.

An (apparently paradoxical) result was proved in [13]: the metric and the affine formulations of General Relativity Theory are always equivalent! More precisely: a generic affine theory (45), when rewritten in terms of the metric and the metric connection $\stackrel{\circ}{\Gamma}$, is equivalent to the conventional Einstein metric theory. This implies an implicit equation which, when solvable, enables us to convert a "minimally coupled to gravity" matter theory (44) into an affine theory for a certain nonmetric connection $\Gamma$. In both cases the nonmetricity tensor

$$
f:=\Gamma-\stackrel{\circ}{\Gamma}
$$

is uniquely defined by the matter fields.

In the present paper we proceed in the opposite direction: we show that the (very troublesome) second order derivatives of the metric, contained in the conventional energy-momentum tensor of the matter, together with the metric Ricci tensor, sum up in a natural way to the (nonmetric) Ricci tensor of a new connection $\Gamma$. This way we obtain a much simpler proof of the above equivalence theorem. But, above all, this result provides a simple physical interpretation of the nonmetric connection. As will be seen in the next section, the transition between the metric and the affine picture consists in gathering the second order derivatives appearing in the Einstein equations (those contained in the metric Ricci tensor and those contained in the matter energy-momentum tensor (1) into a single object: the nonmetric Ricci tensor.

The proof will be carried out in two steps: first, starting with a generic matter Lagrangian $\mathcal{L}_{\mathrm{M}}^{\text {mat }}$ of the conventional metric theory, we show how to simplify the Einstein equations by writing them in the universal form (27) for a certain "affine matter Lagrangian" $\mathcal{L}_{\mathrm{A}}^{\text {mat }}$, in general different from $\mathcal{L}_{\mathrm{M}}^{\text {mat}}$; second, we prove that the functional $\mathcal{L}_{\mathrm{A}}^{\text {mat }}$ constructed in the first step generates the same equations for the matter fields and, therefore, is the correct affine matter Lagrangian. Thus, the Legendre transformation (29) leads directly to the affine formulation of the same theory. Detailed calculations (with examples) are also given in [18].

\section{The simplified form of the Einstein equations}

In this section we analyze in detail the nongeneric properties of the Einstein equations in the standard formulation and propose a remedy for them. We 
begin with a conventional, metric Lagrangian

$$
\mathcal{L}_{\mathrm{M}}=\mathcal{L}_{\mathrm{H}}+\mathcal{L}_{\mathrm{M}}^{\mathrm{mat}}
$$

where $\mathcal{L}_{\mathrm{H}}$ is the Hilbert Lagrangian given by (36) and the matter Lagrangian

$$
\mathcal{L}_{\mathrm{M}}^{\mathrm{mat}}=\mathcal{L}_{\mathrm{M}}^{\mathrm{mat}}(g, \stackrel{\circ}{\Gamma}, \varphi, \partial \varphi)=\mathcal{L}_{\mathrm{M}}^{\mathrm{mat}}(g, \partial g, \varphi, \partial \varphi)
$$

does not belong to the special class (34), which was necessary to apply directly the affine theory. The variation of $\mathcal{L}_{\mathrm{H}}$ with respect to $g_{\mu \nu}$ produces $-\frac{1}{2 k} \stackrel{\mathcal{G}}{ }^{\mu \nu}$ and, therefore, instead of (37), we obtain the following Einstein equations (again, we denote $\partial_{\lambda} g_{\mu \nu}=g_{\mu \nu, \lambda}$ ):

$$
\frac{1}{k} \stackrel{\mathcal{G}}{\mu \nu}^{\mu \nu}=2\left(\frac{\partial \mathcal{L}_{\mathrm{M}}^{\mathrm{mat}}(g, \partial g, \varphi, \partial \varphi)}{\partial g_{\mu \nu}}-\partial_{\lambda} \frac{\partial \mathcal{L}_{\mathrm{M}}^{\mathrm{mat}}(g, \partial g, \varphi, \partial \varphi)}{\partial g_{\mu \nu, \lambda}}\right),
$$

where the quantity on the right-hand side is called "the total metric energymomentum tensor" of the matter. The above form of the Einstein equations displays a weak point of the theory in its metric formulation: the lack of universality. Indeed, we cannot treat the left-hand side as the universal second order differential operator acting on the metric field $g$ and the right hand side as the "source" carried by the matter, because the last term above also contains, in general, the second order derivatives of the metric. This means that, even qualitatively, the properties of the above Einstein equations are not universal and depend heavily on the properties of the matter. In particular, the common belief that the weak perturbations of a solution obey its light cone structure is, in general, false because the second order derivatives of the metric, coming from the right-hand side, may change completely the causal structure carried by the Einstein tensor on the lefthand side.

As will be shown later, the affine formulation eliminates this phenomenon: second order operators acting on the configuration fields $\Gamma$ and $\varphi$ are universal and the interaction between matter and gravity is described by the sources which are of the first differential order. We are going to rewrite the Einstein equations in a form well adapted for this purpose.

Another bad feature of equations (49) is that the splitting of the righthand side into the two terms is not coordinate invariant, but an immediate remedy for this is the standard Palatini trick [20]: we take $g$ and $\stackrel{\circ}{\Gamma}$ as independent variables, instead of $g$ and $\partial g$. Defining

$$
\stackrel{\circ}{\mathcal{J}}_{\lambda}^{\mu \nu}:=\frac{\partial \mathcal{L}_{\mathrm{M}}^{\mathrm{mat}}(g, \stackrel{\circ}{\Gamma}, \varphi, \partial \varphi)}{\partial \stackrel{\Gamma}{ }^{\lambda}{ }_{\mu \nu}}
$$

we may rewrite the Einstein equations (49) in the following, covariant form:

$$
\frac{1}{k} \stackrel{\circ}{\mathcal{G}}^{\mu \nu}=2 \frac{\partial \mathcal{L}_{\mathrm{M}}^{\mathrm{mat}}\left(g, \stackrel{\circ}{\Gamma},_{\varphi}, \partial \varphi\right)}{\partial g_{\mu \nu}}-\stackrel{\circ}{\nabla}_{\beta}\left(2 g^{\alpha(\mu} \stackrel{\circ}{\mathcal{J}}_{\alpha}{ }^{\nu) \beta}-g^{\alpha \beta} \stackrel{\circ}{\mathcal{J}}_{\alpha}{ }^{\mu \nu}\right) .
$$


A simple, algebraic proof of the equivalence between (49) and (51) is contained in Appendix B.1. Further simplification of this equations is obtained if we encode the information about the metric in the density $\pi$, instead of g. Using the same arguments as in formula (38), we obtain the following, equivalent form of the Einstein equations:

$$
\begin{aligned}
& \stackrel{\circ}{K}_{\mu \nu} \\
& =-\frac{\partial \mathcal{L}_{\mathrm{M}}^{\operatorname{mat}}(\pi, \stackrel{\circ}{\Gamma}, \varphi, \partial \varphi)}{\partial \pi^{\mu \nu}}-k \frac{g_{\mu \rho} g_{\nu \sigma}-\frac{1}{2} g_{\mu \nu} g_{\rho \sigma}}{\sqrt{|\operatorname{det} g|}} \stackrel{\circ}{\nabla}_{\beta}\left(2 g^{\alpha(\rho} \stackrel{\circ}{\mathcal{J}}_{\alpha}^{\sigma) \beta}-g^{\alpha \beta} \stackrel{\mathcal{J}}{\alpha}^{\rho \sigma}\right) .
\end{aligned}
$$

If $\mathcal{L}_{\mathrm{M}}^{\text {mat }}$ does not depend on $\stackrel{\circ}{\Gamma}$, we have $\stackrel{\circ}{\mathcal{J}}^{2} 0$ and equations (52) reduce to (27). In this case, the affine and metric principles are equivalent, provided we identify $\mathcal{L}_{\mathrm{M}}^{\text {mat }}$ with $\mathcal{L}_{\mathrm{A}}^{\text {mat }}, \stackrel{\circ}{\Gamma}$ with $\Gamma$ and $\stackrel{\circ}{K}$ with $K$. However, the status of the metricity condition $\nabla g \equiv 0$ changes while passing from $\mathcal{L}_{\mathrm{M}}^{\text {mat }}$ to $\mathcal{L}_{\mathrm{A}}^{\text {mat }}$. In the metric picture this condition is assumed a priori, but in the affine picture it is derived from the variational principle as the field equations for an independent variable $\Gamma$ (cf. the status of equation (14) in the "metric" and the "affine" formulation of the oscillator theory).

Consider now a generic case $\stackrel{\mathcal{J}}{\neq} \neq 0$. No direct identification of the metric and affine variables is possible. However, we are going to incorporate the last " $\stackrel{\circ}{\nabla} \dot{\mathcal{J}}$ " term from the right side of (52), together with $\stackrel{\circ}{K}$ on the left side, into the symmetric part $K$ of the Ricci tensor of a certain nonmetric connection $\Gamma$, which is defined in the sequel. In this way, the conventional Einstein equations (52) will be rewritten in a universal form, analogous to (27):

$$
K_{\mu \nu}=-\frac{\partial \mathcal{L}_{\mathrm{A}}^{\mathrm{mat}}(\pi, \Gamma, \varphi, \partial \varphi)}{\partial \pi^{\mu \nu}}
$$

where the "affine matter Lagrangian" $\mathcal{L}_{\mathrm{A}}^{\text {mat }}$ will be specified in the sequel too.

To prove that such a simplification is possible, consider any symmetric connection $\Gamma$ and its nonmetricity tensor $f$ defined as follows:

$$
f_{\mu \nu}^{\lambda}:=\Gamma_{\mu \nu}^{\lambda}-\stackrel{\circ}{\Gamma}_{\mu \nu}^{\lambda} .
$$

It is a matter of simple calculations (see Appendix B.2) that the symmetric part $K$ of the affine Ricci tensor of $\Gamma$ can be expressed as a sum of the metric Ricci tensor $\stackrel{\circ}{K}$ and the following combination of $f$ and $\stackrel{\circ}{\nabla} f$ :

$$
K_{\mu \nu}=\stackrel{\circ}{K}_{\mu \nu}+\stackrel{\circ}{\nabla}_{\rho} f_{\mu \nu}^{\rho}-\stackrel{\circ}{\nabla}_{(\mu} f_{\nu) \rho}^{\rho}+f_{\sigma \rho}^{\rho} f_{\mu \nu}^{\sigma}-f_{\sigma \mu}^{\rho} f_{\rho \nu}^{\sigma} .
$$

It is easy to check that the differential terms " $\nabla$ $f$ " in the above equations

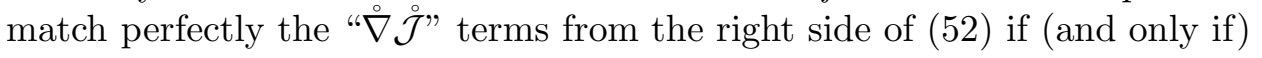


we choose the nonmetricity $f$ as the following, linear function of $\mathcal{J}$ :

$$
\begin{aligned}
f^{\lambda}{ }_{\mu \nu} & :=\frac{k}{\sqrt{|\operatorname{det} g|}}\left[2 \stackrel{\mathcal{J}}{(\mu}_{(\mu}^{\rho \lambda} g_{\nu) \rho}-\stackrel{\mathcal{J}}{\rho}_{\rho}^{\rho \lambda} g_{\mu \nu}-\stackrel{\mathcal{J}}{\rho}_{\rho}{ }^{\sigma \tau} g^{\rho \lambda}\left(g_{\mu \sigma} g_{\nu \tau}-\frac{1}{2} g_{\sigma \tau} g_{\mu \nu}\right)\right. \\
& \left.+\frac{2}{3} \delta_{(\mu}^{\lambda} \dot{\circ}_{\rho}^{\rho \sigma} g_{\nu) \sigma}-\delta_{(\mu}^{\lambda} \stackrel{\circ}{\mathcal{J}}_{\nu)}^{\rho \sigma} g_{\rho \sigma}\right] .
\end{aligned}
$$

To be able to convert (according to (55)) $\stackrel{\circ}{K}$ on the left-hand side of (52) into $K$ and thus to rewrite the Einstein equations in the universal form (153), we need the algebraic terms " $f \cdot f$ " in (55), which are still missing. We are going to prove that they may be easily obtained if we replace the "metric matter Lagrangian" $\mathcal{L}_{\mathrm{M}}^{\text {mat }}$ by the following "affine matter Lagrangian" $\mathcal{L}_{\mathrm{A}}^{\text {mat: }}$

$$
\mathcal{L}_{\mathrm{A}}^{\mathrm{mat}}(\pi, \Gamma, \varphi, \partial \varphi):=\mathcal{L}_{\mathrm{M}}^{\mathrm{mat}}-\pi^{\mu \nu}\left(f_{\sigma \rho}^{\rho} f_{\mu \nu}^{\sigma}-f^{\rho}{ }_{\sigma \mu} f^{\sigma}{ }_{\rho \nu}\right) .
$$

This definition has to be understood as follows. We treat (54) as the implicit equations for $\Gamma$ in terms of $\stackrel{\circ}{\Gamma}$ and the remaining quantities $(\pi, \varphi$ and $\partial \varphi)$ :

$$
\Gamma=\stackrel{\circ}{\Gamma}+f(\pi, \stackrel{\circ}{\Gamma}, \varphi, \partial \varphi),
$$

where $f=f(\pi, \stackrel{\circ}{\Gamma}, \varphi, \partial \varphi)$ is defined by (56) and (50). This yields us an implicit relation $\stackrel{\circ}{\Gamma}=\stackrel{\circ}{\Gamma}(\pi, \Gamma, \varphi, \partial \varphi)$. Using this relation we eliminate $\stackrel{\circ}{\Gamma}$ from $\mathcal{L}_{\mathrm{M}}^{\text {mat }}$ and $f$ on the right side of (57) and, finally, calculate $\mathcal{L}_{\mathrm{A}}^{\text {mat }}$ as a function of $\pi, \Gamma, \varphi$ and $\partial \varphi$.

The following theorem shows that the above procedure leads, indeed, to the universal form (53) of the Einstein equations:

Theorem 1 The Einstein equations (49) (or, equivalently, (52)) generated by the metric matter Lagrangian $\mathcal{L}_{\mathrm{M}}^{\mathrm{mat}}$ are equivalen 4 to the equations

$$
K_{\mu \nu}=-\frac{\partial \mathcal{L}_{\mathrm{A}}^{\mathrm{mat}}(\pi, \Gamma, \varphi, \partial \varphi)}{\partial \pi^{\mu \nu}},
$$

for the symmetric part $K$ of the Ricci tensor of a (possibly nonmetric) connection $\Gamma=\stackrel{\circ}{\Gamma}+f$, where the nonmetricity $f$ is uniquely defined by the metric and the matter fields $\varphi$ via equations (56) and (50). The affine matter Lagrangian $\mathcal{L}_{\mathrm{A}}^{\text {mat }}$ generating this equations is defined by equation (57).

The above statement means that for any solution of the Einstein equations (49), the corresponding (possibly nonmetric) connection $\Gamma$ (defined uniquely by equations (58), (56) and (50) ) satisfies the universal, simplified form (53) of the Einstein equations. The opposite is also true: for any solution of equations (53) generated by a given affine matter Lagrangian $\mathcal{L}_{\mathrm{A}}^{\text {mat }}$,

\footnotetext{
${ }^{4}$ In fact, the validity of this result goes far beyond the generic case, when all the implicit functions considered above are well defined. The nongeneric case may also be treated in terms of symplectic relations 16. The only difference is that additional Lagrangian constraints may arise in the nongeneric case.
} 
the metric connection satisfies the Einstein equations in the conventional form (49) generated by the corresponding metric matter Lagrangian $\mathcal{L}_{\mathrm{M}}^{\text {mat }}$ and related with the former by equation (57). We shall see in the sequel that also the matter equations for $\varphi$, generated respectively by $\mathcal{L}_{\mathrm{M}}^{\text {mat }}$ and $\mathcal{L}_{\mathrm{A}}^{\text {mat }}$, are equivalent.

To conclude this Section we prove Theorem 1. For this purpose we shall write the field equations in terms of the derivatives of $\mathcal{L}_{\mathrm{M}}^{\mathrm{mat}}(\pi, \stackrel{\circ}{\Gamma}, \varphi, \partial \varphi)$ with respect to its arguments. Indeed, we already have

$$
\begin{aligned}
\frac{\partial \mathcal{L}_{\mathrm{M}}^{\text {mat }}}{\partial \pi^{\mu \nu}} & =-\stackrel{\circ}{K}_{\mu \nu}-k \frac{g_{\mu \rho} g_{\nu \sigma}-\frac{1}{2} g_{\mu \nu} g_{\rho \sigma}}{\sqrt{|\operatorname{det} g|}} \stackrel{\circ}{\nabla}_{\beta}\left(2 g^{\alpha(\rho} \stackrel{\circ}{\mathcal{J}}_{\alpha}^{\sigma) \beta}-g^{\alpha \beta} \stackrel{\circ}{\mathcal{J}}_{\alpha} \rho \sigma\right) \\
& =-\left(\stackrel{\circ}{K}_{\mu \nu}+\stackrel{\circ}{\nabla}_{\rho} f_{\mu \nu}^{\rho}-\stackrel{\circ}{\nabla}_{(\mu} f_{\nu) \rho}^{\rho}\right)
\end{aligned}
$$

due to equations (52) and the definition (56) of $f$. Also, $\mathcal{J}$ is equal to $\partial \mathcal{L}_{\mathrm{M}}^{\mathrm{mat}} / \partial \check{\Gamma}^{\circ}$, due to equations (50). Moreover, the Euler-Lagrange equations for the matter fields may be written as $p^{\kappa}{ }_{, \kappa}=\partial \mathcal{L}_{\mathrm{M}}^{\mathrm{mat}} / \partial \varphi$, where

$$
p^{\kappa}:=\partial \mathcal{L}_{\mathrm{M}}^{\operatorname{mat}} / \partial \varphi_{, \kappa}
$$

is the momentum canonically conjugate to the matter fields $\varphi$ and, as usual,

$$
\varphi_{, \kappa}:=\partial_{\kappa} \varphi
$$

and

$$
p_{, \kappa}^{\kappa}:=\partial_{\kappa} p^{\kappa} .
$$

These four field equations (at each space-time point $x$ ) may be written in terms of a single equation imposed on the total differential of $\mathcal{L}_{\mathrm{M}}^{\text {mat }}$, treated as a function of the field variables $\pi^{\mu \nu}, \stackrel{\circ}{\Gamma}^{\lambda} \mu \nu, \varphi$ and $\partial_{\kappa} \varphi$ :

$$
\mathrm{d} \mathcal{L}_{\mathrm{M}}^{\mathrm{mat}}=-\left(\stackrel{\circ}{K}_{\mu \nu}+\stackrel{\circ}{\nabla}_{\rho} f_{\mu \nu}^{\rho}-\stackrel{\circ}{\nabla}_{(\mu} f_{\nu) \rho}^{\rho}\right) \mathrm{d} \pi^{\mu \nu}+\stackrel{\circ}{\mathcal{J}}_{\lambda} \mu \nu \mathrm{d} \stackrel{\circ}{\Gamma}^{\lambda}{ }_{\mu \nu}+p^{\kappa} \mathrm{d} \varphi_{, \kappa}+p^{\kappa}{ }_{, \kappa} \mathrm{d} \varphi .
$$

Using (54) and (57), we may rewrite equivalently this equation in terms of the total differential of the ,improved" Lagrangian $\mathcal{L}_{\mathrm{A}}^{\text {mat: }}$

$$
\begin{aligned}
\mathrm{d} \mathcal{L}_{\mathrm{A}}^{\mathrm{mat}} & =\mathrm{d}\left(\mathcal{L}_{\mathrm{M}}^{\mathrm{mat}}-\pi^{\mu \nu}\left(f_{\rho \sigma}^{\rho} f^{\sigma}{ }_{\mu \nu}-f^{\rho}{ }_{\mu \sigma} f^{\sigma}{ }_{\nu \rho}\right)\right) \\
& =-\left(\stackrel{\circ}{K}_{\mu \nu}+\stackrel{\circ}{\nabla}_{\rho} f_{\mu \nu}^{\rho}-\stackrel{\circ}{\nabla}_{(\mu} f_{\nu) \rho}^{\rho}-f^{\rho}{ }_{\sigma \rho} f_{\mu \nu}^{\sigma}+f^{\rho}{ }_{\sigma \mu} f_{\rho \nu}^{\sigma}\right) \mathrm{d} \pi^{\mu \nu} \\
& -\pi^{\mu \nu} \mathrm{d}\left(f^{\rho}{ }_{\sigma \rho} f^{\sigma}{ }_{\mu \nu}-f^{\rho}{ }_{\sigma \mu} f^{\sigma}{ }_{\rho \nu}\right)+\stackrel{\circ}{\mathcal{J}}_{\lambda}{ }^{\mu \nu} \mathrm{d}\left(\Gamma^{\lambda}{ }_{\mu \nu}-f^{\lambda}{ }_{\mu \nu}\right) \\
& +p^{\kappa} \mathrm{d} \varphi_{, \kappa}+p^{\kappa}{ }_{, \kappa} \mathrm{d} \varphi .
\end{aligned}
$$

To calculate $-\stackrel{\circ}{\mathcal{J}}_{\lambda}{ }^{\mu \nu} \mathrm{d} f^{\lambda}{ }_{\mu \nu}$, we use the equations:

$$
\stackrel{\mathcal{J}}{\lambda}_{\lambda}^{\mu \nu}=2 f_{\rho \lambda}^{(\mu} \pi^{\nu) \rho}-f_{\rho \lambda}^{\rho} \pi^{\mu \nu}-\delta_{\lambda}^{(\mu} f_{\rho \sigma}^{\nu)} \pi^{\rho \sigma},
$$


inverse to (56). This implies:

$$
\begin{aligned}
-\dot{\mathcal{J}}_{\lambda}{ }^{\mu \nu} \mathrm{d} f^{\lambda}{ }_{\mu \nu}= & -\left(f^{\mu}{ }_{\lambda \rho} \pi^{\rho \nu}+f^{\nu}{ }_{\lambda \rho} \pi^{\rho \mu}-f^{\rho}{ }_{\lambda \rho} \pi^{\mu \nu}\right) \mathrm{d} f^{\lambda}{ }_{\mu \nu} \\
& +\left(f^{\nu}{ }_{\kappa \rho} \pi^{\rho \kappa}+f^{\kappa}{ }_{\kappa \rho} \pi^{\nu \rho}-f^{\rho}{ }_{\kappa \rho} \pi^{\nu \kappa}\right) \mathrm{d} f^{\alpha}{ }_{\nu \alpha} \\
= & \pi^{\mu \nu} \mathrm{d}\left(f^{\rho}{ }_{\sigma \rho} f^{\sigma}{ }_{\mu \nu}-f^{\rho}{ }_{\sigma \mu} f^{\sigma}{ }_{\rho \nu}\right) .
\end{aligned}
$$

Inserting this into (61), we get

$$
\begin{aligned}
\mathrm{d} \mathcal{L}_{\mathrm{A}}^{\text {mat }} & =-\left(\stackrel{\circ}{K}_{\mu \nu}+\stackrel{\circ}{\nabla}_{\rho} f_{\mu \nu}^{\rho}-\stackrel{\circ}{\nabla}_{(\mu} f_{\nu) \rho}^{\rho}-f^{\rho}{ }_{\sigma \rho} f^{\sigma}{ }_{\mu \nu}+f^{\rho}{ }_{\sigma \mu} f^{\sigma}{ }_{\rho \nu}\right) \mathrm{d} \pi^{\mu \nu} \\
& +\dot{\mathcal{J}}_{\lambda}{ }^{\mu \nu} \mathrm{d} \Gamma^{\lambda}{ }_{\mu \nu}+p^{\kappa} \mathrm{d} \varphi_{, \kappa}+p^{\kappa}{ }_{, \kappa} \mathrm{d} \varphi .
\end{aligned}
$$

Using formula (55) we see that the terms in the bracket combine to $K_{\mu \nu}$. Hence, we obtain the following, extremely compact formulation of the field equations of the theory (both the gravitational and matter equations), as a single equation imposed on the total differential of $\mathcal{L}_{\mathrm{A}}^{\text {mat: }}$

$$
\mathrm{d} \mathcal{L}_{\mathrm{A}}^{\mathrm{mat}}(\pi, \Gamma, \varphi, \partial \varphi)=-K_{\mu \nu} \mathrm{d} \pi^{\mu \nu}+\stackrel{\mathcal{J}}{\lambda}_{\lambda}^{\mu \nu} \mathrm{d} \Gamma^{\lambda}{ }_{\mu \nu}+p^{\kappa} \mathrm{d} \varphi_{, \kappa}+p^{\kappa}{ }_{, \kappa} \mathrm{d} \varphi .
$$

This completes the proof of Theorem 1 .

\section{The equivalence of the variational principles}

We have shown that one can write the Einstein equations in a much simpler form $K_{\mu \nu}=-\frac{\partial \mathcal{L}_{\mathrm{A}}^{\mathrm{mat}}(\pi, \Gamma, \varphi, \partial \varphi)}{\partial \pi^{\mu \nu}}$. What remains to be shown is that the scalar density $\mathcal{L}_{\mathrm{A}}^{\text {mat }}$ is the affine matter Lagrangian we've been discussing in Section 1. In order to do so, we will show that it fulfills all dynamical equations generated by the "true" affine matter Lagrangian, taken as a function of $\pi, \Gamma, \varphi$ and $\partial \varphi$ variables.

Theorem 2 The solutions of the Einstein equations derived from the metric matter Lagrangian $\mathcal{L}_{\mathrm{M}}^{\mathrm{mat}}(\pi, \stackrel{\circ}{\Gamma}, \varphi, \partial \varphi)$ are equivalent to the solutions of the $d y$ namical equations derived from the affine matter Lagrangian $\mathcal{L}_{\mathrm{A}}^{\text {mat }}(\pi, \Gamma, \varphi, \partial \varphi)$, if and only if

$$
\Gamma_{\mu \nu}^{\lambda}=\stackrel{\circ}{\Gamma}_{\mu \nu}^{\lambda}+f_{\mu \nu}^{\lambda}
$$

and

$$
\begin{aligned}
\mathcal{L}_{\mathrm{A}}^{\mathrm{mat}}(\pi, \Gamma, \varphi, \partial \varphi) & =\mathcal{L}_{\mathrm{M}}^{\mathrm{mat}}(\pi, \stackrel{\circ}{\Gamma}(\pi, \Gamma, \varphi, \partial \varphi), \varphi, \partial \varphi) \\
& -\pi^{\mu \nu}\left(f^{\rho}{ }_{\sigma \rho} f^{\sigma}{ }_{\mu \nu}-f_{\sigma \mu}^{\rho} f^{\sigma}{ }_{\rho \nu}\right),
\end{aligned}
$$

where the nonmetricity tensor $f=f(\pi, \Gamma, \varphi, \partial \varphi)$ is given by the formula

$$
\begin{aligned}
f^{\lambda}{ }_{\mu \nu}= & \frac{k}{\sqrt{|\operatorname{det} g|}}\left[2 \mathcal{J}_{(\mu}{ }^{\rho \lambda} g_{\nu) \rho}-\mathcal{J}_{\rho}{ }^{\rho \lambda} g_{\mu \nu}-\mathcal{J}_{\rho}{ }^{\sigma \tau} g^{\rho \lambda}\left(g_{\mu \sigma} g_{\nu \tau}-\frac{1}{2} g_{\sigma \tau} g_{\mu \nu}\right)\right. \\
& \left.+\frac{2}{3} \delta_{(\mu}^{\lambda} \mathcal{J}_{\rho}^{\rho \sigma} g_{\nu) \sigma}-\delta_{(\mu}^{\lambda} \mathcal{J}_{\nu)}{ }^{\rho \sigma} g_{\rho \sigma}\right]
\end{aligned}
$$


with the nonmetricity current $\mathcal{J}$ defined as

$$
\mathcal{J}_{\lambda}{ }^{\mu \nu}:=\frac{\partial \mathcal{L}_{\mathrm{A}}^{\mathrm{mat}}(\pi, \Gamma, \varphi, \partial \varphi)}{\partial \Gamma^{\lambda}{ }_{\mu \nu}} .
$$

Moreover, the above quantity is numerically equal to its metric analogue: $\mathcal{J}_{\lambda}{ }^{\mu \nu}=\mathcal{J}_{\lambda}{ }^{\mu \nu}$, where the latter is defined by the corresponding metric formula (50).

The proof of the identity $\mathcal{J}_{\lambda}{ }^{\mu \nu}=\dot{\mathcal{J}}_{\lambda}{ }^{\mu \nu}$ follows trivially from the equation (64). Moreover, the above definition of the nonmetricity tensor $f$ implies the following identity (see Appendix B.3), equivalent to equations (47):

$$
\nabla_{\lambda} \pi^{\mu \nu}-\delta_{\lambda}^{(\mu} \nabla_{\kappa} \pi^{\nu) \kappa}=\mathcal{J}_{\lambda}^{\mu \nu}
$$

Hence, the combined system of the metric field equations for the "matter + gravity system", encoded in the equation (64), is equivalent to the following set of equations:

$$
\begin{aligned}
K_{\mu \nu} & =-\frac{\partial \mathcal{L}_{\mathrm{A}}^{\mathrm{mat}}}{\partial \pi^{\mu \nu}}, \\
\nabla_{\lambda} \pi^{\mu \nu}-\delta_{\lambda}^{(\mu} \nabla_{\kappa} \pi^{\nu) \kappa} & =\frac{\partial \mathcal{L}_{\mathrm{A}}^{\mathrm{mat}}}{\partial \Gamma^{\lambda}{ }_{\mu \nu}}, \\
p^{\kappa} & =\frac{\partial \mathcal{L}_{\mathrm{A}}^{\mathrm{mat}}}{\partial \varphi_{, \kappa}}, \\
p^{\kappa}{ }_{, \kappa} & =\frac{\partial \mathcal{L}_{\mathrm{A}}^{\mathrm{mat}}}{\partial \varphi},
\end{aligned}
$$

which are exactly the equations arising from the affine variational principle (see Appendix $\mathrm{A}$ or [1]) for the affine matter Lagrangian $\mathcal{L}_{\mathrm{A}}^{\text {mat }}$.

The argument given above can be reversed, constructing the metric matter Lagrangian from a given affine matter Lagrangian. The definition (56) arises then as the solution of the Euler-Lagrange equations (67) for $\Gamma$. This completes the proof of Theorem 2 ,

Adding to $\mathcal{L}_{\mathrm{A}}^{\text {mat }}$ the term $\pi^{\mu \nu} K_{\mu \nu}$, where we insert the relation $\pi=$ $\pi(K, \Gamma, \phi, \partial \phi)$ given implicitly by equations (66), we define the total affine Lagrangian:

$$
\mathcal{L}_{\mathrm{A}}(K, \Gamma, \varphi, \partial \varphi):=\mathcal{L}_{\mathrm{A}}^{\mathrm{mat}}(\pi, \Gamma, \varphi, \partial \varphi)+\pi^{\mu \nu}(K, \Gamma, \varphi, \partial \varphi) K_{\mu \nu} .
$$

This is a Legendre transformation which converts equation (64) to the following, equivalent equation

$$
\mathrm{d} \mathcal{L}_{\mathrm{A}}(K, \Gamma, \varphi, \partial \varphi)=\pi^{\mu \nu} \mathrm{d} K_{\mu \nu}+\mathcal{J}_{\lambda}{ }^{\mu \nu} \mathrm{d} \Gamma^{\lambda}{ }_{\mu \nu}+p^{\kappa} \mathrm{d} \varphi_{, \kappa}+p^{\kappa}{ }_{,}{ }_{\kappa} \mathrm{d} \varphi .
$$

These are precisely (see Appendix A) the Euler-Lagrange equations derived in the affine formulation of the theory. 
This completes the proof of the equivalence of the metric and the affine principles: each solution of the dynamical equations generated by the metric principle can be transformed into the corresponding solution of the dynamical equations generated by the affine principle.

It is interesting to observe that the numerical value of the total affine Lagrangian is equal to

$$
\begin{aligned}
\mathcal{L}_{\mathrm{A}} & =\mathcal{L}_{\mathrm{M}}^{\mathrm{mat}}+\pi^{\mu \nu}\left(K_{\mu \nu}-f_{\sigma \rho}^{\rho} f_{\mu \nu}^{\sigma}+f_{\sigma \mu}^{\rho} f_{\rho \nu}^{\sigma}\right) \\
& =\mathcal{L}_{\mathrm{M}}+\pi^{\mu \nu}\left(K_{\mu \nu}-\stackrel{\circ}{K}_{\mu \nu}-f^{\rho}{ }_{\sigma \rho} f^{\sigma}{ }_{\mu \nu}+f^{\rho}{ }_{\sigma \mu} f^{\sigma}{ }_{\rho \nu}\right) \\
& =\mathcal{L}_{\mathrm{M}}+\pi^{\mu \nu}\left(\stackrel{\circ}{\nabla}_{\lambda} f^{\lambda}{ }_{\mu \nu}-\stackrel{\circ}{\nabla}_{(\mu} f^{\rho}{ }_{\nu) \rho}\right) \\
& =\mathcal{L}_{\mathrm{M}}+\partial_{\lambda}\left(\pi^{\mu \nu}\left(f^{\lambda}{ }_{\mu \nu}-\delta_{\mu}^{\lambda} f_{\nu \rho}^{\rho}\right)\right),
\end{aligned}
$$

and, whence, differs from the total metric Lagrangian by a complete divergence only (see also [13]).

The transformation from the metric to the affine picture may be summarized as follows: we rewrite the numerical value of $\mathcal{L}_{\mathrm{A}}$ as:

$$
\mathcal{L}_{\mathrm{A}}=\mathcal{L}_{\mathrm{M}}^{\mathrm{mat}}-\pi^{\mu \nu} \frac{\partial \mathcal{L}_{\mathrm{M}}^{\mathrm{mat}}(\pi, \stackrel{\circ}{\Gamma}, \varphi, \partial \varphi)}{\partial \pi^{\mu \nu}} .
$$

Inserting (56) into the equation $\Gamma=\stackrel{\circ}{\Gamma}+f(\pi, \stackrel{\circ}{\Gamma}, \varphi, \partial \varphi)$, and solving the resulting equation with respect to $\Gamma^{\circ}$ (where $\mathcal{J}$ is treated as a function of $\pi, \stackrel{\circ}{\Gamma}, \varphi$ and $\partial \varphi)$, we get the relation $\stackrel{\circ}{\Gamma}=\stackrel{\circ}{\Gamma}(\pi, \Gamma, \varphi, \partial \varphi)$. Inserting this relation into $f=f(\pi, \Gamma, \varphi, \partial \varphi)$ we get $f=f(\pi, \Gamma, \varphi, \partial \varphi)$. Inserting this relation into the modified version (52) of the metric Einstein equations:

$$
K_{\mu \nu}-f_{\mu \nu}^{\rho} f_{\rho \sigma}^{\sigma}+f_{\mu \sigma}^{\rho} f_{\nu \rho}^{\sigma}=-\frac{\partial \mathcal{L}_{\mathrm{M}}^{\operatorname{mat}}(\pi, \stackrel{\circ}{\Gamma}(\pi, \Gamma, \varphi, \partial \varphi), \varphi, \partial \varphi)}{\partial \pi^{\mu \nu}}
$$

we get the relation $K=K(\pi, \Gamma, \varphi, \partial \varphi)$. Inverting this relation with respect to the metric we get $\pi=\pi(K, \Gamma, \varphi, \partial \varphi)$. Inserting this relation into the relation $\stackrel{\circ}{\Gamma}^{\circ} \stackrel{\circ}{\Gamma}(\pi, \Gamma, \varphi, \partial \varphi)$ gives the relation $\stackrel{\circ}{\Gamma}=\stackrel{\circ}{\Gamma}(K, \Gamma, \varphi, \partial \varphi)$. We use these two relations to eliminate the metric quantities $\pi$ and $\stackrel{\circ}{\Gamma}$ from the right-hand side of (73) . This way we obtain $\mathcal{L}_{\mathrm{A}}=\mathcal{L}_{\mathrm{A}}(K, \Gamma, \varphi, \partial \varphi)$ without computing the intermediate quantity $\mathcal{L}_{\mathrm{A}}^{\text {mat }}$.

For the matter Lagrangians obeying the condition (34) (i. e. for the theories considered in [1]), we have $f \equiv 0$ and most of the above relations are trivial: $\Gamma=\stackrel{\circ}{\Gamma}$ and $K=\stackrel{\circ}{K}$. The only relation which remains to be inverted is the Einstein equation $K=K(\pi, \varphi, \partial \varphi)$. The resulting relation $\pi=\pi(K, \varphi, \partial \varphi)$, when inserted into the metric Lagrangian $\mathcal{L}_{\mathrm{M}}$, gives us $\mathcal{L}_{\mathrm{A}}$.

\section{Conclusions}

The affine variational principle leads to such a formulation of the General Relativity theory which is, in some aspects, simpler than the one based on the metric principle. The metric formulation has the following problems: 
1. The Hilbert Lagrangian is of second differential order in $g$, and yet it generates second order (and not 4-th order, as one would expect) equations. This is due to nongeneric cancellations.

2. The status of the momentum canonically conjugate to the metric is obscure: if one takes as the momentum the quantity $\frac{\partial \mathcal{L}_{\mathrm{H}}}{\partial g_{\mu \nu, \lambda \kappa}}$, which is formally correct, one gets second type constraints for the canonical momentum:

$$
\frac{\partial \mathcal{L}_{\mathrm{H}}}{\partial g_{\mu \nu, \lambda \kappa}} \equiv \frac{\sqrt{\mid \operatorname{det} g}}{2 k}\left(g^{\mu \kappa} g^{\nu \lambda}-g^{\mu \nu} g^{\lambda \kappa}\right)
$$

The correct canonical structure (the ADM structure) is obtained only when we perform the symplectic reduction of the theory with respect to the above constraints.

3. As already mentioned, the "sources of gravity", represented in the metric formulation by the energy-momentum tensor, contain not only the first but also the second derivatives of the metric and, therefore, may change completely the causal behaviour of the Einstein equations. The second order term contained in the Einstein tensor cannot be considered anymore as the "principal part" of the equations because of another second order term contained in the metric energy-momentum tensor.

The affine principle is free of these defects. The affine Lagrangian $\mathcal{L}_{\mathrm{A}}$ is of the first differential order in $\Gamma$ and, correctly, generates second order EulerLagrange equations. No additional constraints on the symmetric connection $\Gamma$ are imposed. No total derivatives are "built in" in the Lagrangian, and it is not required to be of any special form. The canonical momentum, given by formula (76), is well defined, transforms like a tensor and represents another geometric quantity describing the geometric structure of space-time: the metric tensor. The canonical structure of the theory, first described by Arnowitt, Deser and Misner in their fundamental paper [17], considerably simplifies in this formulation (see [4] for details). Finally, as shown by Ashtekar-Lewandowski approach to quantum gravity (see [11, 12, 10]), the description of the configuration of the gravitational field in terms of the connection is much more natural. Contrary to the space of metric tensors, the space of connections carries an affine structure and provides a natural arena for path integrals. 


\section{Appendixes}

\section{A The affine variational principle}

In [1] a class of variational principles has been considered, based on the affine, symmetric (not necessarily metric) connection $\Gamma$ as the configuration variable. The Lagrangian was assumed to be an invariant scalar density built from $\Gamma$ and its first partial derivatives $\partial \Gamma$, together with the matter field(s) $\varphi$ and its first partial derivatives:

$$
\mathcal{L}_{\mathrm{A}}=\mathcal{L}_{\mathrm{A}}(\Gamma, \partial \Gamma, \varphi, \partial \varphi) .
$$

Defining the canonical momenta: conjugate to $\Gamma$

$$
\pi_{\lambda}{ }^{\mu \nu \kappa}:=\frac{\partial \mathcal{L}_{\mathrm{A}}(\Gamma, \partial \Gamma, \varphi, \partial \varphi)}{\partial \Gamma^{\lambda}{ }_{\mu \nu, \kappa}},
$$

where $\Gamma_{\mu \nu, \kappa}^{\lambda}=\partial_{\kappa} \Gamma_{\mu \nu}^{\lambda}$, and conjugate to $\varphi$

$$
p^{\kappa}:=\frac{\partial \mathcal{L}_{\mathrm{A}}(\Gamma, \partial \Gamma, \varphi, \partial \varphi)}{\partial \varphi_{, \kappa}},
$$

we calculate the variation of the Lagrangian as follows:

$$
\begin{aligned}
& \mathrm{d} \mathcal{L}_{\mathrm{A}}=\pi_{\lambda}{ }^{\mu \nu \kappa} \mathrm{d} \Gamma^{\lambda}{ }_{\mu \nu, \kappa}+\frac{\partial \mathcal{L}_{\mathrm{A}}}{\partial \Gamma^{\lambda}{ }_{\mu \nu}} \mathrm{d} \Gamma^{\lambda}{ }_{\mu \nu}+p^{\kappa} \mathrm{d} \varphi_{, \kappa}+\frac{\partial \mathcal{L}_{\mathrm{A}}}{\partial \varphi} \mathrm{d} \varphi \\
& =\left(\frac{\partial \mathcal{L}_{\mathrm{A}}}{\partial \Gamma^{\lambda}{ }_{\mu \nu}}-\partial_{\kappa} \pi_{\lambda}{ }^{\mu \nu \kappa}\right) \mathrm{d} \Gamma^{\lambda}{ }_{\mu \nu}+\left(\frac{\partial \mathcal{L}_{\mathrm{A}}}{\partial \varphi}-\partial_{\kappa} p^{\kappa}\right) \mathrm{d} \varphi \\
& +\partial_{\kappa}\left(\pi_{\lambda}{ }^{\mu \nu \kappa} \mathrm{d} \Gamma^{\lambda}{ }_{\mu \nu}+p^{\kappa} \mathrm{d} \varphi\right) \text {. }
\end{aligned}
$$

The Euler-Lagrange equations of the theory:

$$
\begin{aligned}
\partial_{\kappa} \pi_{\lambda}{ }^{\mu \nu \kappa} & =\frac{\partial \mathcal{L}_{\mathrm{A}}(\Gamma, \partial \Gamma, \varphi, \partial \varphi)}{\partial \Gamma^{\lambda}{ }_{\mu \nu}} \\
\partial_{\kappa} p^{\kappa} & =\frac{\partial \mathcal{L}_{\mathrm{A}}(\Gamma, \partial \Gamma, \varphi, \partial \varphi)}{\partial \varphi}
\end{aligned}
$$

mean that the volume part of the variation must vanish or, equivalently, the entire variation $\mathrm{d} \mathcal{L}_{\mathrm{A}}$ reduces to the boundary part:

$$
\begin{aligned}
\mathrm{d} \mathcal{L}_{\mathrm{A}}= & \partial_{\kappa}\left(\pi_{\lambda}{ }^{\mu \nu \kappa} \mathrm{d} \Gamma^{\lambda}{ }_{\mu \nu}+p^{\kappa} \mathrm{d} \varphi\right) \\
= & \pi_{\lambda}{ }^{\mu \nu \kappa} \mathrm{d} \Gamma^{\lambda}{ }_{\mu \nu, \kappa}+\left(\partial_{\kappa} \pi_{\lambda}{ }^{\mu \nu \kappa}\right) \mathrm{d} \Gamma^{\lambda}{ }_{\mu \nu} \\
& +p^{\kappa} \mathrm{d} \varphi_{, \kappa}+\left(\partial_{\kappa} p^{\kappa}\right) \mathrm{d} \varphi .
\end{aligned}
$$

To obtain a theory which is invariant with respect to diffeomorphisms we assume that $\mathcal{L}_{\mathrm{A}}$ depends upon the derivatives of $\Gamma$ via the only invariant 
quantity which one may construct: the Riemann curvature tensor $R_{\mu \nu \kappa}^{\lambda}$. Lagrangians of the type $\mathcal{L}_{\mathrm{A}}=\mathcal{L}_{\mathrm{A}}(\Gamma, R, \varphi, \partial \varphi)$ describe a much broader class of theories than the General Relativity theory (see [21, 22]), i. e. the resulting field equations are not necessarily equivalent to the Einstein equations. However, it has been proved in [1] that we can reduce this freedom to exactly Einstein's theory by postulating a specific dependence of $\mathcal{L}_{\mathrm{A}}$ upon the Riemann tensor: the only information about the curvature which the Lagrangian is sensitive to is the symmetric part $K_{\mu \nu}=\frac{1}{2}\left(R_{\mu \nu}+R_{\nu \mu}\right)$ of its Ricci tensor $R_{\mu \nu}=R_{\mu \lambda \nu}^{\lambda}$ (unlike in the case of the metric connection, the Ricci tensor is not necessarily symmetric):

$$
\mathcal{L}_{\mathrm{A}}=\mathcal{L}_{\mathrm{A}}(\Gamma, K, \varphi, \partial \varphi) .
$$

Hence, the variation (78) of the Lagrangian reduces to

$$
\mathrm{d} \mathcal{L}_{\mathrm{A}}=\pi^{\mu \nu} \mathrm{d} K_{\mu \nu}+\mathcal{J}_{\lambda}^{\mu \nu} \mathrm{d} \Gamma^{\lambda}{ }_{\mu \nu}+p^{\kappa} \mathrm{d} \varphi_{, \kappa}+\frac{\partial \mathcal{L}_{\mathrm{A}}}{\partial \varphi} \mathrm{d} \varphi
$$

where we have introduced the following tensor densities:

$$
\pi^{\mu \nu}:=\frac{\partial \mathcal{L}_{\mathrm{A}}(\Gamma, K, \varphi, \partial \varphi)}{\partial K_{\mu \nu}},
$$

and

$$
\mathcal{J}_{\lambda}{ }^{\mu \nu}:=\frac{\partial \mathcal{L}_{\mathrm{A}}(\Gamma, K, \varphi, \partial \varphi)}{\partial \Gamma^{\lambda}{ }_{\mu \nu}} .
$$

However, $K$ is linear in $\partial \Gamma$ and quadratic in $\Gamma$ :

$$
K_{\mu \nu}=\Gamma_{\mu \nu, \lambda}^{\lambda}-\Gamma_{\lambda(\mu, \nu)}^{\lambda}+\Gamma_{\alpha \lambda}^{\lambda} \Gamma_{\mu \nu}^{\alpha}-\Gamma_{\alpha \nu}^{\lambda} \Gamma_{\mu \lambda}^{\alpha} .
$$

Hence, the first term in (83) may be calculated as follows:

$$
\begin{aligned}
\pi^{\mu \nu} \mathrm{d} K_{\mu \nu} & =\left(\delta_{\lambda}^{\kappa} \pi^{\mu \nu}-\delta_{\lambda}^{(\mu} \pi^{\nu) \kappa}\right) \mathrm{d} \Gamma_{\mu \nu, \kappa}^{\lambda} \\
& +\left(\pi^{\mu \nu} \Gamma^{\kappa}{ }_{\lambda \kappa}+\pi^{\sigma \kappa} \Gamma_{\sigma \kappa}^{\mu} \delta_{\lambda}^{\nu}-2 \pi^{\sigma \nu} \Gamma_{\sigma \lambda}^{\mu}\right) \mathrm{d} \Gamma^{\lambda}{ }_{\mu \nu} .
\end{aligned}
$$

Comparing it with (81) we obtain from (83):

$$
\pi_{\lambda}^{\mu \nu \kappa}=\delta_{\lambda}^{\kappa} \pi^{\mu \nu}-\delta_{\lambda}^{(\mu} \pi^{\nu) \kappa} .
$$

Taking into account that $\pi$ is a tensor density, it is easy to check that (87) contains the difference between the partial and the covariant divergence:

$$
\pi^{\mu \nu} \Gamma_{\lambda \kappa}^{\kappa}+\pi^{\sigma \kappa} \Gamma_{\sigma \kappa}^{\mu} \delta_{\lambda}^{\nu}-2 \pi^{\sigma \nu} \Gamma_{\sigma \lambda}^{\mu}=\partial_{\kappa} \pi_{\lambda}^{\mu \nu \kappa}-\nabla_{\kappa} \pi_{\lambda}{ }^{\mu \nu \kappa} .
$$

Combining these results, the variation (83) reduces to

$$
\begin{aligned}
\mathrm{d} \mathcal{L}_{\mathrm{A}} & =\left(\mathcal{J}_{\lambda}{ }^{\mu \nu}-\nabla_{\kappa} \pi_{\lambda}{ }^{\mu \nu \kappa}\right) \mathrm{d} \Gamma^{\lambda}{ }_{\mu \nu}+\left(\frac{\partial \mathcal{L}_{\mathrm{A}}}{\partial \varphi}-\partial_{\kappa} p^{\kappa}\right) \mathrm{d} \varphi \\
& +\partial_{\kappa}\left(\pi_{\lambda}{ }^{\mu \nu \kappa} \mathrm{d} \Gamma^{\lambda}{ }_{\mu \nu}+p^{\kappa} \mathrm{d} \varphi\right) .
\end{aligned}
$$


The Euler-Lagrange equations mean that the volume part of (90) vanishes:

$$
\nabla_{\kappa} \pi_{\lambda}{ }^{\mu \nu \kappa}=\nabla_{\lambda} \pi^{\mu \nu}-\delta_{\lambda}^{(\mu} \nabla_{\rho} \pi^{\nu) \rho}=\mathcal{J}_{\lambda}{ }^{\mu \nu}, \quad p^{\kappa}{ }_{, \kappa}=\frac{\partial \mathcal{L}_{\mathrm{A}}}{\partial \varphi} .
$$

We interpret $\pi$ as the contravariant density of the metric:

$$
\pi^{\mu \nu}=: \frac{\sqrt{|\operatorname{det} g|}}{2 k} g^{\mu \nu} .
$$

The formula $\pi_{\rho}^{\mu \nu \rho}=4 \pi^{\mu \nu}-\pi^{\mu \nu}$ or, equivalently,

$$
\pi^{\mu \nu}=\frac{1}{3} \pi_{\rho}^{\mu \nu \rho}
$$

gives a one-to-one correspondence between $\pi_{\lambda}{ }^{\mu \nu \kappa}$ and $\pi^{\mu \nu}$. Taking the trace of the first equation of (191), we obtain the relation $\mathcal{J}_{\rho}^{\mu \rho}=\nabla_{\rho} \pi^{\mu \rho}-\frac{5}{2} \nabla_{\rho} \pi^{\mu \rho}$, which leads to an equivalent form of the Euler-Lagrange equations generated by $\mathcal{L}_{\mathrm{A}}$ :

$$
\nabla_{\lambda} \pi^{\mu \nu}=\mathcal{J}_{\lambda}{ }^{\mu \nu}-\frac{2}{3} \delta_{\lambda}^{(\mu} \mathcal{J}_{\rho}{ }^{\nu) \rho} \quad p^{\kappa}{ }_{, \kappa}=\frac{\partial \mathcal{L}_{\mathrm{A}}(\Gamma, \partial \Gamma, \varphi, \partial \varphi)}{\partial \varphi}
$$

If $\mathcal{L}_{\mathrm{A}}=\mathcal{L}_{\mathrm{A}}(K, \varphi, \partial \varphi)$ (which was assumed in the paper [1]) then, according to (85), $\mathcal{J}=0$ and the gravitational part of these equations becomes

$$
\nabla_{\lambda} \pi^{\mu \nu}=0
$$

The only solution $\Gamma$ of this equations is the metric (Levi-Civitta) connection: $\Gamma=\stackrel{\circ}{\Gamma}$. In a generic case, the only solution of the "nonmetricity equations" $\nabla_{\lambda} \pi^{\mu \nu}=\mathcal{J}_{\lambda}{ }^{\mu \nu}-\frac{2}{3} \delta_{\lambda}^{(\mu} \mathcal{J}_{\rho}{ }^{\nu) \rho}$ is the following connection:

$$
\Gamma:=\stackrel{\circ}{\Gamma}+f,
$$

where the "nonmetricity tensor" $f$ is uniquely defined in terms of the "nonmetricity current" $\mathcal{J}$ by formula (56) (see Appendix B.3 for the proof).

To analyze the relation of the above "affine theory" with the conventional metric Einstein theory it is useful to subtract from the Lagrangian $\mathcal{L}_{\mathrm{A}}$ the generalized Hilbert term $\mathcal{L}_{\mathrm{H}}$, given by the scalar curvature:

$$
\mathcal{L}_{\mathrm{H}}=\frac{\sqrt{|\operatorname{det} g|}}{2 k} R=\frac{\sqrt{|\operatorname{det} g|}}{2 k} g^{\mu \nu} R_{\mu \nu}=\pi^{\mu \nu} K_{\mu \nu} .
$$

This way we define the "affine matter Lagrangian":

$$
\begin{aligned}
\mathcal{L}_{\mathrm{A}}^{\mathrm{mat}}(\pi, \Gamma, \varphi, \partial \varphi): & =\mathcal{L}_{\mathrm{A}}(\Gamma, K(\pi, \Gamma, \varphi, \partial \varphi), \varphi, \partial \varphi) \\
& -\pi^{\mu \nu} K_{\mu \nu}(\pi, \Gamma, \varphi, \partial \varphi)
\end{aligned}
$$

The above transformation may be interpreted as the Legendre transformation between $\pi^{\mu \nu}$ and $K_{\mu \nu}$. To rewrite the field equations of the theory 
in terms of $\mathcal{L}_{\mathrm{A}}^{\text {mat }}$, we use the standard technique of the Legendre transformation [16, 23]. For this purpose we write the field equations (91) together with the definitions (77) and (84) of the momenta in the form of a generating relation imposed on the total derivative of $\mathcal{L}_{\mathrm{A}}$ :

$$
\begin{aligned}
\mathrm{d} \mathcal{L}_{\mathrm{A}}(K, \Gamma, \varphi, \partial \varphi) & =\pi^{\mu \nu} \mathrm{d} K_{\mu \nu}+p^{\kappa} \mathrm{d} \varphi_{, \kappa}+p^{\kappa}{ }_{, \kappa} \mathrm{d} \varphi \\
& +\left(\nabla_{\lambda} \pi^{\mu \nu}-\delta_{\lambda}^{(\mu} \nabla_{\rho} \pi^{\nu) \rho}\right) \mathrm{d} \Gamma^{\lambda}{ }_{\mu \nu} .
\end{aligned}
$$

This implies the following equation for the total differential of the function (96):

$$
\begin{aligned}
& \mathrm{d} \mathcal{L}_{\mathrm{A}}^{\mathrm{mat}}(\pi, \Gamma, \varphi, \partial \varphi)=-K_{\mu \nu} \mathrm{d} \pi^{\mu \nu}+\left(\nabla_{\lambda} \pi^{\mu \nu}-\delta_{\lambda}^{(\mu} \nabla_{\rho} \pi^{\nu) \rho}\right) \mathrm{d} \Gamma^{\lambda}{ }_{\mu \nu} \\
& \quad+p^{\kappa} \mathrm{d} \varphi_{, \kappa}+p^{\kappa}{ }_{, \kappa} \mathrm{d} \varphi
\end{aligned}
$$

or, equivalently:

$$
\begin{aligned}
K_{\mu \nu} & =-\frac{\partial \mathcal{L}_{\mathrm{A}}^{\mathrm{mat}}(\pi, \Gamma, \varphi, \partial \varphi)}{\partial \pi^{\mu \nu}} \\
\mathcal{J}_{\lambda}{ }^{\mu \nu} & =\nabla_{\lambda} \pi^{\mu \nu}-\delta_{\lambda}^{(\mu} \nabla_{\rho} \pi^{\nu) \rho} \\
p^{\kappa}{ }_{, \kappa} & =\frac{\partial \mathcal{L}_{\mathrm{A}}^{\text {mat }}(\pi, \Gamma, \varphi, \partial \varphi)}{\partial \varphi}
\end{aligned}
$$

where the quantities $\mathcal{J}$ and $p$ are now defined as

$$
\mathcal{J}_{\lambda}^{\mu \nu}:=\frac{\partial \mathcal{L}_{\mathrm{A}}^{\mathrm{mat}}(\pi, \Gamma, \varphi, \partial \varphi)}{\partial \Gamma^{\lambda}{ }_{\mu \nu}}
$$

and

$$
p^{\kappa}:=\frac{\partial \mathcal{L}_{\mathrm{A}}^{\mathrm{mat}}(\pi, \Gamma, \varphi, \partial \varphi)}{\partial \varphi_{, \kappa}} .
$$

Of course, the equivalence between equations (99) and (94) may be proved directly $\sqrt{5}$ by calculating the derivatives of $\mathcal{L}_{\mathrm{A}}^{\text {mat }}$ defined by $(96)$.

We will now show that the first equations of (99) may be rewritten in the conventional form:

$$
\mathcal{G}^{\mu \nu}=k \mathcal{T}^{\mu \nu},
$$

where the ,,affine energy-momentum tensor density" $\mathcal{T}$ is defined as follows:

$$
\mathcal{T}^{\mu \nu}=2 \frac{\partial \mathcal{L}_{\mathrm{A}}^{\mathrm{mat}}(g, \Gamma, \varphi, \partial \varphi)}{\partial g_{\mu \nu}},
$$

\footnotetext{
${ }^{5}$ The proof based on formula (98) uses, in fact, symplectic relations. It is valid also when there are Lagrangian constraints imposed on the configuration variables. In such a theory the "response" of the system is not unique: the relation $K=K(\pi, \Gamma, \varphi, \partial \varphi)$ may be noninvertible, but still formula (96) implies the field equations (99) (see 16, 23] for the details).
} 
and

$$
\mathcal{G}^{\mu \nu}:=\sqrt{|\operatorname{det} g|}\left(K^{\mu \nu}-\frac{1}{2} g^{\mu \nu} R\right)
$$

is the density of the Einstein tensor constructed from $K$. Indeed, according to (92), the metric tensor $g$ can be reconstructed from $\pi$ as

$$
g_{\mu \nu}=2 k \sqrt{|\operatorname{det} \pi|}\left(\pi^{-1}\right)_{\mu \nu} .
$$

Inserting (104) into the first equations of (99), we get

$$
\begin{aligned}
K_{\mu \nu}= & -\frac{\partial g_{\rho \sigma}}{\partial \pi^{\mu \nu}} \frac{\partial \mathcal{L}_{\mathrm{A}}^{\mathrm{mat}}(g, \Gamma, \varphi, \partial \varphi)}{\partial g_{\rho \sigma}} \\
= & 2 k \sqrt{|\operatorname{det} \pi|}\left(\left(\pi^{-1}\right)_{\rho(\mu}\left(\pi^{-1}\right)_{\nu) \sigma}-\frac{1}{2}\left(\pi^{-1}\right)_{\mu \nu}\left(\pi^{-1}\right)_{\rho \sigma}\right) \\
& \times \frac{\partial \mathcal{L}_{\mathrm{A}}^{\operatorname{mat}}(g, \Gamma, \varphi, \partial \varphi)}{\partial g_{\rho \sigma}} \\
= & \frac{2 k}{\sqrt{|\operatorname{det} g|}}\left(g_{\rho(\mu} g_{\nu) \sigma}-\frac{1}{2} g_{\mu \nu} g_{\rho \sigma}\right) \frac{\partial \mathcal{L}_{\mathrm{A}}^{\operatorname{mat}}(g, \Gamma, \varphi, \partial \varphi)}{\partial g_{\rho \sigma}}
\end{aligned}
$$

Inverting this equations we obtain (102).

In the paper 24] generalized conservation laws for the energy-momentum tensor (103) (matching the generalized Bianchi identities for the nonmetric Einstein tensor $\mathcal{G}$ ) were analyzed. In the special case of $\mathcal{J}=0$ we have $\Gamma=\stackrel{\circ}{\Gamma}$ and $\mathcal{T}$ reduces to the standard (metric) energy-momentum tensor, provided we identify $\mathcal{L}_{\mathrm{A}}^{\text {mat }}$ with the (metric) matter Lagrangian $\mathcal{L}_{\mathrm{M}}^{\text {mat }}$. Moreover, (103) become the conventional Einstein equations for a matter Lagrangian independent of $\partial g$ :

$$
\stackrel{\mathcal{G}}{\mu}^{\mu \nu}=2 k \frac{\partial \mathcal{L}_{\mathrm{M}}^{\mathrm{mat}}(g, \varphi, \partial \varphi)}{\partial g_{\mu \nu}} .
$$

In this way the equivalence between the affine and the metric approaches was proved in [1] in the special case $\mathcal{J}=0$. The only difference between the two principles in this case is that in the affine approach, equations (105) are postulated (by the specific choice of the Lagrangian) and the metricity equation is a consequence of the Euler-Lagrange equations (94), while in the metric approach the metricity is postulated and (105) (or (84)) is a consequence of the Euler-Lagrange equations.

For the purposes of canonical relativity, let us observe that the field dynamics, written in terms of equation (80), may be further reduced. Indeed, the identities (88) imply:

$$
\partial_{\kappa}\left(\pi_{\lambda}^{\mu \nu \kappa} \mathrm{d} \Gamma_{\mu \nu}^{\lambda}\right)=\partial_{\lambda}\left(\pi^{\mu \nu} \mathrm{d} A_{\mu \nu}^{\lambda}\right),
$$

where we have denoted

$$
A_{\mu \nu}^{\lambda}:=\Gamma_{\mu \nu}^{\lambda}-\delta_{(\mu}^{\lambda} \Gamma_{\nu) \kappa}^{\kappa} .
$$


Hence, equation (80) may be written as follows:

$$
\mathrm{d} \mathcal{L}_{\mathrm{A}}=\partial_{\lambda}\left(\pi^{\mu \nu} \mathrm{d} A_{\mu \nu}^{\lambda}+p^{\lambda} \mathrm{d} \varphi\right)
$$

Integrating it over a four-dimensional volume $\mathcal{O}$ we obtain

$$
\mathrm{d} \int_{\mathcal{O}} \mathcal{L}_{\mathrm{A}}=\int_{\partial \mathcal{O}} \pi^{\mu \nu} \mathrm{d} A_{\mu \nu}^{\perp}+p^{\perp} \mathrm{d} \varphi
$$

It turns out that not all among the 10 components $A_{\mu \nu}^{\perp}$ transversal to the three-surface $\partial \mathcal{O}$ are independent. It was proved in [4] that the four components $A_{\mu \perp}^{\perp}$ may be calculated in terms of the 6 components $A_{k l}^{\perp}$, where $k$ and $l$ label the three coordinates on the boundary. Reducing the right side of (109) with respect to these constraints, we obtain the following form of dynamics:

$$
\mathrm{d} \int_{\mathcal{O}} \mathcal{L}_{\mathrm{A}}=-\frac{1}{16 \pi} \int_{\partial \mathcal{O}} g_{k l} \delta \Pi^{k l}+\int_{\partial \mathcal{O}} p^{\perp} \mathrm{d} \varphi
$$

where $g_{k l}$ is the three-metric of $\mathcal{O}$ and $\Pi^{k l}$ denotes its external curvature in the ADM representation (see [4]). Suppose that $\mathcal{O}=V \times\left[t_{1}, t_{2}\right]$, where $V$ is three-dimensional and spatial, and that $\left[t_{1}, t_{2}\right]$ is the time interval. Then, the boundary $\partial \mathcal{O}$ splits into the space-like part composed of two surfaces $\Sigma_{i}=V \times\left\{t_{i}\right\}$ and the time-like world-tube $T=\partial V \times\left[t_{1}, t_{2}\right]$. The integration of (110) over the Cauchy surfaces $\Sigma_{i}$ gives just the well known ADM canonical structure. But the remaining integral over $T$ captures all the boundary terms which are necessary for the definition of the gravitational Hamiltonian. Moreover, there is also a Dirac-delta-like contribution of the external curvature corresponding to the corners $\partial V \times\left\{t_{i}\right\}$. This contribution modifies the ADM canonical structure within the closed volume $V$ and makes it gauge invariant (see again [4])

\section{B Proofs of various formulas}

\section{B.1 The proof of formula (51)}

The matter Lagrangian depends upon the metric via the metric itself and the metric connection coefficients:

$$
\begin{aligned}
& \frac{\partial \mathcal{L}_{\mathrm{M}}^{\mathrm{mat}}(g, \partial g, \varphi, \partial \varphi)}{\partial g_{\mu \nu}} \\
& =\frac{\partial \mathcal{L}_{\mathrm{M}}^{\mathrm{mat}}(g, \stackrel{\circ}{\Gamma}, \varphi, \partial \varphi)}{\partial g_{\mu \nu}}+\frac{\partial \stackrel{\circ}{\Gamma}^{\alpha}{ }_{\beta \gamma}}{\partial g_{\mu \nu}} \frac{\mathcal{L}_{\mathrm{M}}^{\mathrm{mat}}\left(g, \stackrel{\circ}{\Gamma},_{\varphi}, \partial \varphi\right)}{\partial \Gamma^{\alpha}{ }_{\beta \gamma}} \\
& =\frac{\partial \mathcal{L}_{\mathrm{M}}^{\mathrm{mat}}(g, \stackrel{\circ}{\Gamma}, \varphi, \partial \varphi)}{\partial g_{\mu \nu}}-\frac{1}{2} g^{\alpha(\mu} g^{\nu) \delta}\left(g_{\delta \beta, \gamma}+g_{\delta \gamma, \beta}-g_{\beta \gamma, \delta}\right) \stackrel{\circ}{\mathcal{J}}_{\alpha}^{\beta \gamma} \\
& =\frac{\partial \mathcal{L}_{\mathrm{M}}^{\mathrm{mat}}(g, \stackrel{\circ}{\Gamma}, \varphi, \partial \varphi)}{\partial g_{\mu \nu}}-g^{\alpha\left(\mu \stackrel{\circ}{\Gamma}^{\nu)}\right.}{ }_{\beta \gamma} \stackrel{\circ}{\mathcal{J}}_{\alpha}^{\beta \gamma}
\end{aligned}
$$


where the tensor density $\mathcal{J}$ defined by

$$
\stackrel{\circ}{\mathcal{J}}_{\lambda}^{\mu \nu}:=\frac{\partial \mathcal{L}_{\mathrm{M}}^{\mathrm{mat}}\left(g, \stackrel{\circ}{\Gamma}^{\prime}, \varphi, \partial \varphi\right)}{\partial \Gamma^{\lambda}{ }_{\mu \nu}}
$$

is numerically equal to the nonmetricity current $\mathcal{J}$ in the affine formulation, see formula (64).

The second term on the right side of (51) may also be rewritten along these lines:

$$
\begin{aligned}
& \partial_{\lambda} \frac{\partial \mathcal{L}_{\mathrm{M}}^{\mathrm{mat}}(g, \partial g, \varphi, \partial \varphi)}{\partial g_{\mu \nu, \lambda}}=\partial_{\lambda} \frac{\partial \stackrel{\circ}{\Gamma}^{\alpha}{ }_{\mu \nu}}{\partial g_{\mu \nu, \lambda}} \frac{\partial \mathcal{L}_{\mathrm{M}}^{\mathrm{mat}}(g, \stackrel{\circ}{\Gamma}, \varphi, \partial \varphi)}{\partial \Gamma^{\alpha}{ }_{\beta \gamma}} \\
& =\frac{1}{2}\left[2 g^{\alpha(\mu} \partial_{\gamma} \stackrel{\circ}{\mathcal{J}}_{\alpha}{ }^{\nu) \gamma}-g^{\alpha \gamma} \partial_{\gamma} \stackrel{\mathcal{J}}{\alpha}^{\mu \nu}\right. \\
& \left.-\left(2 g^{\alpha \rho} g^{\sigma(\mu} \stackrel{\circ}{\mathcal{J}}_{\alpha}{ }^{\nu) \gamma}-g^{\alpha \rho} g^{\gamma \sigma} \stackrel{\circ}{\mathcal{J}}_{\alpha}{ }^{\mu \nu}\right) g_{\rho \sigma, \gamma}\right] \\
& =\frac{1}{2}\left[2 g^{\alpha(\mu} \partial_{\beta} \stackrel{\circ}{\mathcal{J}}_{\alpha}{ }^{\nu) \beta}-g^{\alpha \beta} \partial_{\beta} \stackrel{\circ}{\mathcal{J}}_{\alpha}{ }^{\mu \nu}-2 g^{\alpha \beta} \stackrel{\circ}{\Gamma}^{(\mu}{ }_{\beta \gamma} \stackrel{\circ}{\mathcal{J}}_{\alpha}{ }^{\nu) \gamma}\right. \\
& \left.-2 \stackrel{\circ}{\Gamma}^{\beta}{ }_{\alpha \gamma} g^{\alpha(\mu} \stackrel{\circ}{\mathcal{J}}_{\beta}{ }^{\nu) \gamma}+g^{\alpha \beta} \stackrel{\circ}{\Gamma}_{\beta \gamma}^{\gamma} \stackrel{\circ}{\mathcal{J}}_{\alpha}{ }^{\mu \nu}+g^{\alpha \beta} \stackrel{\circ}{\Gamma}^{\gamma}{ }_{\alpha \beta} \stackrel{\circ}{\mathcal{J}}_{\gamma}{ }^{\mu \nu}\right] \text {, }
\end{aligned}
$$

where we use the formula

$$
\stackrel{\circ}{\Gamma}_{\beta \gamma}^{\alpha}:=\frac{1}{2} g^{\alpha \delta}\left(g_{\delta \beta, \gamma}+g_{\delta \gamma, \beta}-g_{\beta \gamma, \delta}\right)
$$

and the identity $0=\stackrel{\circ}{\nabla}_{\lambda} g_{\rho \sigma}=g_{\rho \sigma, \lambda}-\stackrel{\circ}{\Gamma}_{\rho \gamma}^{\tau} g_{\tau \sigma}-\stackrel{\circ}{\Gamma}_{\sigma \gamma}^{\tau} g_{\rho \tau}$. Inserting (111) and (113) into (49) and combining the partial derivatives $\partial \dot{\mathcal{J}}$ together with the products " $\mathcal{J} \cdot \stackrel{\circ}{\Gamma}$ " into the covariant derivatives $\stackrel{\circ}{\nabla} \mathcal{J}^{\circ}$, we get (51).

\section{B.2 The proof of formula (55)}

The symmetric part of the Ricci tensor of the affine connection $\Gamma$ is given by:

$$
K_{\mu \nu}=\partial_{\rho} \Gamma_{\mu \nu}^{\rho}-\partial_{(\mu} \Gamma_{\nu) \rho}^{\rho}+\Gamma_{\sigma \rho}^{\rho} \Gamma_{\mu \nu}^{\sigma}-\Gamma_{\sigma \mu}^{\rho} \Gamma_{\rho \nu}^{\sigma} .
$$

Inserting the decomposition $\Gamma=\stackrel{\circ}{\Gamma}+f$, we get

$$
\begin{aligned}
& K_{\mu \nu}=\overbrace{\partial_{\rho} \stackrel{\circ}{\Gamma}_{\mu \nu}^{\rho}-\partial_{(\mu} \stackrel{\circ}{\Gamma}_{\nu) \rho}^{\rho}+\stackrel{\circ}{\Gamma}^{\rho}{ }_{\sigma \rho} \stackrel{\circ}{\Gamma}^{\sigma}{ }_{\mu \nu}-\stackrel{\circ}{\Gamma}^{\rho}{ }_{\sigma \mu} \stackrel{\circ}{\Gamma}^{\sigma}{ }_{\rho \nu}}^{\stackrel{\circ}{K}_{\mu \nu}} \\
& +\overbrace{\partial_{\rho} f^{\rho}{ }_{\mu \nu}+\stackrel{\circ}{\Gamma}_{\rho \sigma}^{\rho} f^{\sigma}{ }_{\mu \nu}-2{\stackrel{\circ}{\Gamma^{\sigma}}}_{\rho(\mu} f^{\rho}{ }_{\nu) \sigma}}^{\dot{\circ}_{\rho} f^{\rho}{ }_{\mu \nu}} \\
& -\overbrace{\partial_{(\mu} f^{\rho}{ }_{\nu) \rho}+\dot{\Gamma}^{\sigma}{ }_{\mu \nu} f^{\rho}{ }_{\rho \sigma}}^{\nabla_{(\mu} f^{\rho}{ }_{\nu) \rho}}+f^{\rho}{ }_{\sigma \rho} f^{\sigma}{ }_{\mu \nu}-f_{\sigma \mu}^{\rho} f^{\sigma}{ }_{\rho \nu} \\
& =\stackrel{\circ}{K}_{\mu \nu}+\stackrel{\circ}{\nabla}_{\rho} f_{\mu \nu}^{\rho}-\stackrel{\circ}{\nabla}_{(\mu} f_{\nu) \rho}^{\rho}+f_{\sigma \rho}^{\rho} f_{\mu \nu}^{\sigma}-f_{\sigma \mu}^{\rho} f_{\rho \nu}^{\sigma} \text {, }
\end{aligned}
$$

which proves (55). 


\section{B.3 The proof of formula (65)}

We will show that $\Gamma=\stackrel{\circ}{\Gamma}+f$, with the nonmetricity tensor $f$ given by formula (56), is the only solution of the nonmetricity equations (65). As was already mentioned in Appendix $\mathrm{A}$ (cf. the equations (91) and (94)), the equations (65) are equivalent to

$$
\nabla_{\lambda} \pi^{\mu \nu}=\mathcal{J}_{\lambda}^{\mu \nu}-\frac{2}{3} \delta_{\lambda}^{(\mu} \mathcal{J}_{\rho}^{\nu) \rho}
$$

Plugging $\Gamma=\stackrel{\circ}{\Gamma}+f$ into these equations we get

$$
\nabla_{\lambda} \pi^{\mu \nu}=f_{\lambda \rho}^{\mu} \pi^{\nu \rho}+f_{\lambda \rho}^{\nu} \pi^{\mu \rho}-f_{\lambda \rho}^{\rho} \pi^{\mu \nu},
$$

because the remaining terms combine to the covariant derivative $\stackrel{\circ}{\nabla}_{\lambda} \pi^{\mu \nu}$, which vanishes identically.

Contracting both sides with $\pi_{\mu \nu}=g_{\mu \rho} g_{\nu \sigma} \pi^{\rho \sigma}$ gives

$$
\pi_{\mu \nu} \nabla_{\lambda} \pi^{\mu \nu}=-2(2 k \sqrt{|\operatorname{det} g|})^{2} f_{\lambda \rho}^{\rho},
$$

which allows us to rewrite (116) as

$$
f_{\lambda \rho}^{\mu} \pi^{\nu \rho}+f_{\lambda \rho}^{\nu} \pi^{\mu \rho}=\nabla_{\lambda} \pi^{\mu \nu}-\frac{\pi_{\rho \sigma} \pi^{\mu \nu} \nabla_{\lambda} \pi^{\rho \sigma}}{2(2 k \sqrt{|\operatorname{det} g|})^{2}} .
$$

Consider the following quantity:

$$
A_{\lambda}{ }^{\mu \nu}:=\frac{1}{2 k \sqrt{|\operatorname{det} \pi|}}\left(\nabla_{\lambda} \pi^{\mu \nu}-\frac{\pi_{\rho \sigma} \pi^{\mu \nu} \nabla_{\lambda} \pi^{\rho \sigma}}{2(2 k \sqrt{|\operatorname{det} g|})^{2}}\right) .
$$

Raising the lower index we obtain the following identities:

$$
\begin{aligned}
A^{\lambda \mu \nu} & =f^{\mu \nu \lambda}+f^{\nu \mu \lambda} \\
A^{\mu \lambda \nu} & =f^{\lambda \mu \nu}+f^{\nu \mu \lambda} \\
-A^{\nu \mu \lambda} & =-f^{\mu \nu \lambda}-f^{\lambda \nu \mu} .
\end{aligned}
$$

Similarly as in the standard derivation of the formula for the Christoffel symbols, we add them up. After lowering one index we obtain:

$$
f_{\mu \nu}^{\lambda}=A_{(\mu}^{\lambda \rho} g_{\nu) \rho}-\frac{1}{2} g_{\mu \rho} g_{\nu \sigma} g^{\lambda \tau} A_{\tau}^{\rho \sigma} .
$$

Inserting (119) into (121), we get

$$
\begin{aligned}
f_{\mu \nu}^{\lambda} & =\frac{2 k}{\sqrt{|\operatorname{det} g|}}\left[g_{\rho(\mu} \nabla_{\nu)} \pi^{\lambda \rho}-\frac{1}{2} \delta_{(\mu}^{\lambda} g_{\rho \sigma} \nabla_{\nu)} \pi^{\rho \sigma}\right. \\
& \left.-\frac{1}{2}\left(g_{\mu \rho} g_{\nu \sigma} g^{\lambda \tau} \nabla_{\tau} \pi^{\rho \sigma}-\frac{1}{2} g_{\mu \nu} g_{\rho \sigma} g^{\lambda \tau} \nabla_{\lambda} \pi^{\rho \sigma}\right)\right] .
\end{aligned}
$$

Inserting (115) into (122) gives (56), proving that it is the only solution of (65). 


\section{An example of the Legendre transformation}

We have provided an example of the Legendre transformation between $\mathcal{L}_{\mathrm{M}}^{\text {mat }}$ and $\mathcal{L}_{\mathrm{A}}^{\text {mat }}$.

Consider the following metric matter Lagrangian $\mathcal{L}_{\mathrm{M}}^{\text {mat }}$ :

$$
\begin{aligned}
\mathcal{L}_{\mathrm{M}}^{\mathrm{mat}}(g, \stackrel{\circ}{\Gamma}, \phi, \partial \phi) & =\sqrt{|\operatorname{det} g|} \stackrel{\circ}{\nabla}_{(\mu} \phi_{\nu)} \stackrel{\circ}{\nabla}^{(\mu} \phi^{\nu)} \\
& =\sqrt{|\operatorname{det} g|} g^{\mu \lambda} g^{\nu \kappa} \stackrel{\circ}{\nabla}_{(\mu} \phi_{\nu)} \stackrel{\circ}{\nabla}_{(\lambda} \phi_{\kappa)},
\end{aligned}
$$

where $\phi_{\nu}$ is a covariant matter field (using the anti-symmetrized part of $\stackrel{\circ}{\nabla} \phi$ leads to the Maxwell Lagrangian (31) for the electromagnetic field $F_{\mu \nu}=\partial_{\mu} A_{\nu}-\partial_{\nu} A_{\mu}$, see [15]). We are going to carry out the Legendre transformation between $\mathcal{L}_{\mathrm{M}}^{\text {mat }}$ and $\mathcal{L}_{\mathrm{A}}^{\text {mat }}$.

The nonmetricity current $\mathcal{J}$ is equal to

$$
\begin{aligned}
\dot{\mathcal{J}}_{\lambda}^{\mu \nu} & =\frac{\partial \stackrel{\circ}{\nabla}_{\alpha} \phi_{\beta}}{\partial \stackrel{\Gamma}{\Gamma}^{\lambda} \mu \nu} \frac{\partial \mathcal{L}_{\mathrm{M}}^{\mathrm{mat}}}{\partial \dot{\nabla}_{\alpha} \phi_{\beta}} \\
& =-2 \sqrt{|\operatorname{det} g|} g^{\alpha \rho} g^{\beta \sigma} \stackrel{\circ}{\nabla}_{(\rho} \phi_{\sigma)} \delta_{\alpha}^{(\mu} \delta_{\beta}^{\nu)} \phi_{\lambda} \\
& =-2 \sqrt{|\operatorname{det} g|} g^{\mu \rho} g^{\nu \sigma} \stackrel{\circ}{\nabla}_{(\rho} \phi_{\sigma)} \phi_{\lambda} .
\end{aligned}
$$

The corresponding nonmetricity tensor $f$ is, according to the formula (56), equal to

$$
\begin{aligned}
f_{\mu \nu}^{\lambda} & =-2 k\left[g^{\rho \lambda} \phi_{\mu} \stackrel{\circ}{\nabla}_{(\nu} \phi_{\rho)}+g^{\rho \lambda} \phi_{\nu} \stackrel{\circ}{\nabla}_{(\mu} \phi_{\rho)}-g^{\rho \alpha} g^{\lambda \beta} g_{\mu \nu} \phi_{\rho} \stackrel{\circ}{\nabla}_{(\alpha} \phi_{\beta)}\right. \\
& -g^{\rho \lambda} \phi_{\rho} \stackrel{\circ}{\nabla}_{(\mu} \phi_{\nu)}+\frac{1}{2} g^{\alpha \beta} g^{\rho \lambda} g_{\mu \nu} \phi_{\rho} \stackrel{\circ}{\nabla}_{(\alpha} \phi_{\beta)}+\frac{1}{3} \delta_{\mu}^{\lambda} g^{\rho \alpha} \phi_{\rho} \stackrel{\circ}{\nabla}_{(\nu} \phi_{\alpha)} \\
& \left.+\frac{1}{3} \delta_{\nu}^{\lambda} g^{\rho \alpha} \phi_{\rho} \stackrel{\circ}{\nabla}_{(\mu} \phi_{\alpha)}-\frac{1}{2} \delta_{\mu}^{\lambda} \phi_{\nu} g^{\alpha \beta} \stackrel{\circ}{\nabla}_{(\alpha} \phi_{\beta)}-\frac{1}{2} \delta_{\nu}^{\lambda} \phi_{\mu} g^{\alpha \beta} \stackrel{\circ}{\nabla}_{(\alpha} \phi_{\beta)}\right] .
\end{aligned}
$$

To perform the Legendre transformation from $\mathcal{L}_{\mathrm{M}}^{\text {mat }}$ to $\mathcal{L}_{\mathrm{A}}^{\text {mat }}$, we must express $\stackrel{\circ}{\Gamma}$ as a function of $\Gamma$ and the matter field, according to (125). This is an extremely difficult task. However, all we need is to compute $\mathcal{L}_{\mathrm{A}}^{\text {mat }}$ and to express the field configurations in terms of the connection $\Gamma$. For this purpose we have to compute the quadratic combinations of $f$ 's which give the difference between $\mathcal{L}_{\mathrm{M}}^{\text {mat }}$ and $\mathcal{L}_{\mathrm{A}}^{\text {mat }}$ and to express $\stackrel{\circ}{\nabla} \phi^{\circ}$ as a combination of $\nabla \phi$ (and possibly other) terms. The affine covariant derivative of $\phi$ is equal to

$$
\nabla_{\mu} \phi_{\nu}=\stackrel{\circ}{\nabla}_{\mu} \phi_{\nu}-f_{\mu \nu}^{\lambda} \phi_{\lambda}
$$

Inserting (125) into (126) gives

$$
\begin{aligned}
\nabla_{\mu} \phi_{\nu} & =\stackrel{\circ}{\nabla}_{\mu} \phi_{\nu}-2 k\left[\frac{4}{3} g^{\rho \sigma} \phi_{\rho} \phi_{\mu} \stackrel{\circ}{\nabla}_{(\nu} \phi_{\sigma)}+\frac{4}{3} g^{\rho \sigma} \phi_{\rho} \phi_{\nu} \stackrel{\circ}{\nabla}_{(\mu} \phi_{\sigma)}\right. \\
& \left.-\frac{1}{2} g^{\rho \sigma} g^{\tau \eta} g_{\mu \nu} \phi_{\rho} \phi_{\tau} \stackrel{\circ}{\nabla}_{(\sigma} \phi_{\eta)}-g^{\rho \sigma} \phi_{\rho} \phi_{\sigma} \stackrel{\circ}{\nabla}_{(\mu} \phi_{\nu)}-g^{\rho \sigma} \phi_{\mu} \phi_{\nu} \stackrel{\circ}{\nabla}_{(\rho} \phi_{\sigma)}\right] .
\end{aligned}
$$


Correspondingly, we express the relation $\nabla^{\circ} \phi=\stackrel{\circ}{\nabla}(g, \phi, \nabla \phi)$ as a sum (with unknown coefficients) of all possible 2-covariant (with two lower indices and no higher indices) expressions linear in $\nabla \phi$, which could be constructed algebraically from $g, \phi$ and $\nabla \phi$ :

$$
\begin{aligned}
\stackrel{\circ}{\nabla}_{\mu} \phi_{\nu} & =\alpha_{1} \nabla_{(\mu} \phi_{\nu)}+\alpha_{2} \nabla_{[\mu} \phi_{\nu]}+\beta g_{\mu \nu}+\gamma_{1} g^{\rho \sigma} \phi_{\rho} \phi_{\mu}\left(\nabla_{(\nu} \phi_{\sigma)}\right) \\
& +\gamma_{2} g^{\rho \sigma} \phi_{\rho} \phi_{\mu}\left(\nabla_{[\nu} \phi_{\sigma]}\right)+\delta_{1} g^{\rho \sigma} \phi_{\rho} \phi_{\nu}\left(\nabla_{(\mu} \phi_{\sigma)}\right)+\delta_{2} g^{\rho \sigma} \phi_{\rho} \phi_{\nu}\left(\nabla_{[\mu} \phi_{\sigma]}\right) \\
& +\epsilon \phi_{\mu} \phi_{\nu}
\end{aligned}
$$

where lower Greek letters denote the scalar coefficients which do not depend on $\nabla \phi$, with the exception of $\beta$ and $\epsilon$, which are linear in $\nabla \phi$. We shall find their values by plugging this Ansatz into (127), expecting that all terms other than $\nabla_{\mu} \phi_{\nu}$ cancel, and solving the resulting system of linear equations. This system reads:

$$
\begin{aligned}
& 1=(1+2 \psi) \alpha_{1} \\
& 1=\alpha_{2} \\
& 0=k \Omega^{\prime} \alpha_{1}+(1+3 \psi) \beta+\psi \Omega^{\prime} \gamma_{1}+\psi \Omega^{\prime} \delta_{1}+\frac{\psi^{2}}{k} \epsilon \\
& 0=-\frac{8}{3} k \alpha_{1}+\left(1-\frac{1}{3} \psi\right) \gamma_{1}-\frac{1}{3} \psi \delta_{1} \\
& 0=-\frac{8}{3} k \alpha_{1}-\frac{1}{3} \psi \gamma_{1}+\left(1-\frac{1}{3} \psi\right) \delta_{1} \\
& 0=\left(1-\frac{1}{3} \psi\right) \gamma_{2}-\frac{1}{3} \psi \delta_{2} \\
& 0=-\frac{1}{3} \psi \gamma_{2}+\left(1-\frac{1}{3} \psi\right) \delta_{2} \\
& 0=k \Theta^{\prime} \alpha_{1}-\frac{4}{3} k \beta-\frac{5}{3} k \Omega^{\prime} \gamma_{1}-\frac{5}{3} k \Omega^{\prime} \delta_{1}+\left(1-\frac{7}{3} \psi\right) \epsilon,
\end{aligned}
$$

where a scalar invariant $\psi$, independent of the connection, is defined as:

$$
\psi:=k g^{\alpha \beta} \phi_{\alpha} \phi_{\beta}
$$

and

$$
\begin{aligned}
& \Psi^{\prime}:=g^{\mu \rho} g^{\nu \sigma} \nabla_{(\mu} \phi_{\nu)} \nabla_{(\rho} \phi_{\sigma)} \\
& \Omega^{\prime}:=g^{\mu \rho} g^{\nu \sigma} \phi_{\mu} \phi_{\nu} \nabla_{(\rho} \phi_{\sigma)} \\
& \Pi^{\prime}:=g^{\mu \rho} g^{\nu \kappa} g^{\sigma \tau} \phi_{\kappa} \phi_{\tau} \nabla_{(\mu} \phi_{\nu)} \nabla_{(\rho} \phi_{\sigma)} \\
& \Theta^{\prime}:=g^{\mu \nu} \nabla_{(\mu} \phi_{\nu)}
\end{aligned}
$$

are the scalar invariants of the $\Gamma$ connection (their counterparts depending on the metric connection are denoted in the same way, but without a prime). 
The solution of the system reads:

$$
\begin{aligned}
\alpha_{1} & =\frac{1}{1+2 \psi} \\
\alpha_{2} & =1 \\
\beta & =\frac{3 \Theta^{\prime} \psi^{2}-3 k \Omega^{\prime}+23 k \psi \Omega^{\prime}}{(1+2 \psi)\left(3+2 \psi-17 \psi^{2}\right)} \\
\gamma_{1} & =\delta_{1}=-\frac{8 k}{(1+2 \psi)(3-2 \psi)} \\
\gamma_{2} & =\delta_{2}=0 \\
\epsilon & =-\frac{9 k \Theta^{\prime}+21 k \Theta^{\prime} \psi-18 k \Theta^{\prime} \psi^{2}+92 k^{2} \Omega^{\prime}+168 k^{2} \psi \Omega^{\prime}}{(3-2 \psi)(1+2 \psi)\left(3+2 \psi-17 \psi^{2}\right)} .
\end{aligned}
$$

One should note that for certain configurations, some of the coefficients diverge. It does not mean that our procedure is ill-defined in this case, it merely means that we have some additional constrainst imposed on $\nabla \phi$ (once again, we refer to [16]).

Inserting the Ansatz (128), with the coefficients given by (130), into (123) gives

$$
\begin{aligned}
\mathcal{L}_{\mathrm{M}}^{\mathrm{mat}}(g, \phi, \nabla \phi) & =\sqrt{|\operatorname{det} g|}\left[\alpha_{1}^{2} \Psi^{\prime}+4 \beta^{2}+\frac{\psi^{2}}{k^{2}} \epsilon^{2}+2\left(2 \alpha_{1}+\frac{\psi}{k} \gamma_{1}\right) \gamma_{1} \Pi^{\prime}\right. \\
& \left.+\left(2 \alpha_{1} \epsilon+4 \beta \gamma_{1}+4 \frac{\psi}{k} \gamma_{1} \epsilon\right) \Omega^{\prime}+2 \alpha_{1} \beta \Theta^{\prime}+2 \frac{\psi}{k} \gamma_{1}^{2} \Omega^{\prime 2}\right]
\end{aligned}
$$

Calculating the term $\pi^{\mu \nu}\left(f^{\rho}{ }_{\mu \nu} f^{\sigma}{ }_{\rho} \rho \sigma-f^{\rho}{ }_{\mu \sigma} f^{\sigma}{ }_{\nu \rho}\right)$ (subtracted from $\mathcal{L}_{\mathrm{M}}^{\mathrm{mat}}$ in order to get $\mathcal{L}_{\mathrm{A}}^{\text {mat }}$ ) in terms of $\nabla \phi$ and substituting (128) gives, after subtracting it from the density (131) and substituting (130), the final result:

$$
\begin{aligned}
\mathcal{L}_{\mathrm{A}}^{\mathrm{mat}}(g, \phi, \nabla \phi) & =\sqrt{|\operatorname{det} g|}\left(C_{\Psi^{\prime}} \Psi^{\prime}+C_{\Pi^{\prime}} \Pi^{\prime}+C_{\Theta^{\prime 2}} \Theta^{\prime 2}\right. \\
& \left.+C_{\Omega^{\prime} \Theta^{\prime}} \Omega^{\prime} \Theta^{\prime}+C_{\Omega^{\prime 2}} \Omega^{\prime 2}\right)
\end{aligned}
$$

where

$$
\begin{aligned}
C_{\Psi^{\prime}}= & \frac{1-2 k \psi}{1+2 \psi}, \quad C_{\Pi^{\prime}}=\frac{64 k \psi^{2}+256 k^{3} \psi^{2}+64 k^{2} \psi(3+2 \psi)-144}{9+12 \psi-12 \psi^{2}} \\
C_{\Theta^{\prime 2}}= & \frac{3 k^{3} \psi^{3}(1+3 \psi)^{2}+6 \psi^{2}\left(3+2 \psi-11 \psi^{2}\right)}{(1+2 \psi)^{2}\left(3+2 \psi-17 \psi^{2}\right)} \\
& +\frac{3 k^{2} \psi^{2}\left(9+40 \psi+21 \psi^{2}-54 \psi^{3}\right)}{(1+2 \psi)^{2}\left(3+2 \psi-17 \psi^{2}\right)} \\
& +\frac{\psi k\left(9+12 \psi-116 \psi^{2}-122 \psi^{3}+256 \psi^{4}\right)}{(1+2 \psi)^{2}\left(3+2 \psi-17 \psi^{2}\right)}
\end{aligned}
$$




$$
\begin{aligned}
C_{\Omega^{\prime} \Theta^{\prime}}= & \frac{8 \psi\left(k+4 k^{2}\right)-12 k}{(3-2 \psi)(1+2 \psi)^{2}} \\
& +\frac{108 k-18 k \psi(14+15 k)+12 k \psi^{2}\left(1+46 k+88 k^{2}\right)}{(3-2 \psi)(1+2 \psi)^{2}\left(3+2 \psi-17 \psi^{2}\right)^{2}} \\
& +\frac{2 k \psi^{3}\left(170+665 k-2580 k^{2}-92 k^{3}\right)}{(3-2 \psi)(1+2 \psi)^{2}\left(3+2 \psi-17 \psi^{2}\right)^{2}} \\
& -\frac{2 k \psi^{4}\left(1180-2358 k+2276 k^{2}+444 k^{3}\right)}{(3-2 \psi)(1+2 \psi)^{2}\left(3+2 \psi-17 \psi^{2}\right)^{2}} \\
& -\frac{16 k \psi^{5}\left(34+277 k+429 k^{2}-63 k^{3}\right)}{(3-2 \psi)(1+2 \psi)^{2}\left(3+2 \psi-17 \psi^{2}\right)^{2}}, \\
C_{\Theta^{\prime 2}}= & \frac{1620 k^{2}-216 k^{2} \psi(243-80 k)+12 k^{2} \psi^{2}\left(6115+7118 k+1748 k^{2}\right)}{3(3-2 \psi)^{2}(1+2 \psi)^{2}\left(3+2 \psi-17 \psi^{2}\right)^{2}} \\
& +\frac{8 k^{2} \psi^{3}\left(16300-17125 k+25120 k^{2}+1058 k^{3}\right)}{3(3-2 \psi)^{2}(1+2 \psi)^{2}\left(3+2 \psi-17 \psi^{2}\right)^{2}} \\
& -\frac{16 k^{2} \psi^{4}\left(7403+29604 k-11204 k^{2}-1932 k^{3}\right)}{3(3-2 \psi)^{2}(1+2 \psi)^{2}\left(3+2 \psi-17 \psi^{2}\right)^{2}} \\
& +\frac{96 k^{2} \psi^{5}\left(646+1433 k-2240 k^{2}+294 k^{3}\right)}{3(3-2 \psi)^{2}(1+2 \psi)^{2}\left(3+2 \psi-17 \psi^{2}\right)^{2}} .
\end{aligned}
$$

The transformation of $\mathcal{L}_{\mathrm{A}}^{\text {mat }}$ to $\mathcal{L}_{\mathrm{A}}$ consists in solving a nonlinear (because of the terms like $\psi^{3}, \psi^{4}$ and $\psi^{5}$ ) problem.

Remark: The transformation between $\mathcal{L}_{\mathrm{A}}^{\text {mat }}$ and $\mathcal{L}_{\mathrm{M}}^{\text {mat }}$ is practical only for the Lagrangians quadratic in the covariant derivatives, since only then we have a system of linear equations to solve. We also want to mention that for such Lagrangians, our transformation is of higher order. That is, taking $\nabla \phi, \stackrel{\circ}{\nabla} \phi$ and $\phi$ itself to be of the first order in $\phi$, we have

$$
\nabla \phi-\stackrel{\circ}{\nabla} \phi=O\left(\phi^{3}\right) \text {. }
$$

The proof is easy: the nonmetricity tensor is proportional to the derivative of $\nabla \phi \nabla \phi$ over $\Gamma$, hence it is of the order $O\left(\phi^{2}\right)$. Thus, the difference $\nabla \phi-\nabla^{\circ} \phi=$ " $f \cdot \phi$ " is of the order $O\left(\phi^{3}\right)$.

\section{References}

[1] J. Kijowski: Gen. Rel. Grav. 9, 857 (1978).

[2] J. D. Finley and J. F. Plebański: J. Math. Phys. 17, 585 (1976).

[3] E. T. Newman: Gen. Rel. Grav. 7, 107 (1976).

[4] J. Kijowski: Gen. Rel. Grav. 29, 307 (1997). 
[5] L. B. Szabados: Quasi-local energy-momentum and angular momentum in GR: A review article, Living Reviews in Relativity, http://relativity.livingreviews.org/ (2004).

[6] P. T. Chruściel: Recent results in mathematical gravity, in: Proceedings of the Conference GR17, Dublin, July 2004, arXiv:gr-qc/0411008

[7] E. Czuchry, Jezierski, J, and J. Kijowski: Phys. Rev. D 70, 124010 (2004).

[8] J. Jezierski, J. Kijowski, and E. Czuchry: Phys. Rev. D 65, 064036 (2002).

[9] J. Kijowski, G. Magli and D. Malafarina: Phys. Rev. D 74084017 (2006)

[10] A. Ashtekar and J. Lewandowski: Class. Quant. Grav. 21, R53 (2004).

[11] A. Ashtekar: Lectures on Non-perturbative Canonical Gravity, World Scientific, Singapore 1991, notes prepared in collaboration with R. S. Tate.

[12] A. Ashtekar and J. Lewandowski: Quantum field theory of geometry, in: The Proceedings of the Conference on Historical Examination and Philosophical Reflections on the Foundations of Quantum Field Theory, "Conceptual foundations of quantum field theory", Boston 1996, 187206.

[13] M. Ferraris and J. Kijowski: Rend. Sem. Mat. Univ. Polit. Torino 41, 169 (1983).

[14] J. Kijowski and G. Magli: Class. Quant. Grav. 15, 3891 (1998).

[15] M. Ferraris and J. Kijowski: Lett. Math. Phys. 5, 127 (1981).

[16] J. Kijowski and W. M. Tulczyjew: A Symplectic Framework for Field Theories, vol. 107 of LNP, Springer, Berlin-New York 1979.

[17] R. Arnowitt, S. Deser, and C. W. Misner: The dynamics of General Relativity, in: L. Witten (ed.), Gravitation: an Introduction to Current Research, Wiley, New York 1962, 227-265.

[18] R. Werpachowski: Master's thesis, Cardinal Stefan Wyszyński University in Warsaw, 2003.

[19] J. Kijowski, B. Pawlik, and W. Tulczyjew: Bull. Acad. Polon. Sci. (math., phys., astr.) 27, 163 (1979).

[20] A. Palatini: Rend. Circ. Mat. Palermo 43, 203 (1919). 
[21] M. Ferraris and J. Kijowski: Gen. Rel. Grav. 14, 37 (1982).

[22] M. Ferraris and J. Kijowski: Gen. Rel. Grav. 14, 165 (1982).

[23] P. Chruściel, J. Jezierski, and J. Kijowski: Hamiltonian Field Theory in the Radiating Regime, vol. 70 of LNP Monographs, Springer, BerlinNew York 2002.

[24] M. Francaviglia and J. Kijowski: Gen. Rel. Grav. 12, 279 (1980). 